Aufsatz

Ulrich Blum/Inéz Labucay*

\title{
Governance vulnerabler strategischer Wertschöpfungsketten im Zeichen der Deglobalisierung
}

https://doi.org/10.1515/sirius-2021-3004

Kurzfassung: Fragmentierte Wertschöpfungsketten haben in den vergangenen Jahrzehnten die globale Wohlstandsverteilung bestimmt, zugleich aber Unternehmen und Volkswirtschaften aufgrund ihrer Vernetztheit gerade im Kern ihrer industriellen Basis einer hohen strategischen Vulnerabilität ausgesetzt. Ein Beispiel sind Seltene Erden, die für eine Vielzahl an Hightech- und Greentech-Produkten (Elektromobilität, Windräder), aber auch für traditionelle Industrien wie Automobil- und Elektroindustrie (Halbleiter) und für den Verteidigungssektor (Präzisionslenkwaffen) funktionskritisch sind. Vulnerabilität ist hier deshalb hoch, da kaum Substitutionsmöglichkeiten bestehen und Recycling noch am Anfang steht. Rohstoffe und Technologien sind somit ins Zentrum strategischer Industrie- und Handelspolitik gerückt und das Gestalten und nach Möglichkeit das Dominieren globaler Wertschöpfungsketten gewinnen an Bedeutung. Der Zugang $\mathrm{zu}$ Wertschöpfungsketten wird zum Kristallisationspunkt wirtschafts- und sicherheitspolitischer Strategien. Der Beitrag analysiert die strategische Vulnerabilität unterschiedlicher Markt-Wettbewerbskonstellationen und prüft Abwehr- und Gestaltungsmaßnahmen für eine Governance von Vulnerabilität. Anhand von Hochleistungsmagneten und dem zugrundeliegenden Materialstrom der Seltenen Erden werden konkrete Rohstoffstrategien exemplarisch aufgezeigt und die zunehmend geforderte Rohstoff- und Technologiesouveränität kritisch diskutiert, was angesichts der wirtschafts- und sicherheitspolitischen Dimen-

Die Verfasser danken der Fraunhofer-Gesellschaft und dem Ministerium für Wirtschaft, Wissenschaft und Digitalisierung des Landes Sachsen-Anhalt für die Förderung des Projekts (Bewilligungsnummer I 108)

*Kontakt: Prof. Dr. Dr. h. c. Ulrich Blum, Gründungsdirektor, Center for Economics of Materials, Martin-Luther-Universität HalleWittenberg, E-Mail: ulrich.blum@wiwi.uni-halle.de

Inéz Labucay, Martin-Luther-Universität Halle-Wittenberg,

E-Mail: inez.labucay@wiwi.uni-halle.de sion Seltener Erden und regulativem Druck zur Umsetzung von Lieferketten-Compliance und Circular Economy dringlich erscheint.

Schlüsselwörter: Kritikalität, Rohstoff, Vulnerabilität, Wertschöpfungskette

Abstract: Global wealth distribution has been decisively hinging on participation in increasingly fragmented value chains in the last decades. At the same time, their interconnectedness has made companies and national economies prone to vulnerability of their core industrial base. The dependency on rare earth elements which are crucial for the functioning of high-performance magnets built in hightech and greentech products (e. g., electromobility, wind turbines) but also crucial for more traditional sectors like automotive and electronics (e. g., semiconductors) and for the defense industry (e. g., precision guidance ammunitions) poses strategic challenges as there is hardly any substitution to date with recycling activities still in their infancy. Resources and technologies have thus come to the fore of strategic industrial and trade policy with a crucial role accruing to the shaping and possibly the dominance of global value chains. Access to global value chains has become the focal point of economic and security policy strategy making. The paper analyzes the interplay between market structure, competition and strategic vulnerability of global value chains. Based on this, governance strategies to safeguard against vulnerability are discussed. De-risking of global value chains appears to be mandatory with respect to technological and resource sovereignty, especially in the light of rare earth elements being highly relevant for both economic and security policy and with regulatory pressure on the rise to increase accountability across value chains and to move towards the Circular Economy.

Keywords: criticality, resources, vulnerability, value chain 


\section{Einleitung - strategische Vulnera- bilität von Wertschöpfungsketten}

Bis Mitte des Jahres 2021 standen in vielen Automobilwerken in Europa und Asien aufgrund von Lieferengpässen bei Halbleitern die Produktionslinien still. Mit verantwortlich war das SARS-CoV-2-(„Corona“) Virus, das seit Anfang 2020 die Welt in Atem hält und nachdrücklich zeigt, wie verwundbar Volkswirtschaften gegenüber unvorbereitet auftretenden Schocks sind (Abbildung 1). Der internationale Handel mit Gütern, der schon in den vergangenen Jahren deutlich zurückgegangen ist, nimmt weiter ab eine Ausnahme macht nur China, welches das Virus durch eine strikte Lockdown- und Abschottungsstrategie inzwischen weitgehend eliminiert hat.

\begin{tabular}{ll}
\hline Branche & Grad der Betroffenheit \\
\hline Verarbeitendes Gewerbe & Gastgewerbe \\
Sonstige wirtschaftliche Dienstleistungen \\
Quandel, Instandhaltung, Reparatur von KfZ
\end{tabular}

Abb. 1: Vulnerabilität einzelner Branchen durch die Corona-Pandemie

Quelle: eigene Darstellung in Anlehnung Bundesagentur für Arbeit (2020), 24; Bild des Virus: https://phil.cdc.gov/details.aspx?pid= 23312 (Alissa Eckert, Dan Higgins); Vulnerabilität gemessen anhand des Rückgangs sozialversicherungspflichtiger Beschäftigung (Top 5-Rückgänge), Stand August 2020

Die Vulnerabilität ist aber auch auf stark fragmentierte und schwer kontrollierbare Wertschöpfungsketten zurückzuführen. So dominiert bei der Chipproduktion Auftragsfertigung durch zunehmend verhandlungsmächtige Lieferanten. Allerdings ist auch in der Eurozone bereits ein umgekehrter Trend der Deglobalisierung von Wertschöpfungsketten $\mathrm{zu}$ beobachten, offensichtlich ist die Risikoausgleichsfunktion von Märkten verloren gegangen. ${ }^{1}$ Sieht man von einer kurzen Erholung ab, so kann die Weltfinanzkrise 2008/2009 als Trendumkehr der Globalisierung angesehen werden. Erstmalig wuchs im Jahr 2016 der Welthandel schwächer als die Weltproduktion, das Ausmaß der Globalisierung war damit so gering wie zuletzt vor 30 Jahren, wie Abbildung 2 verdeutlicht.

1 Blum 2018, 738 u. 740.
Aber auch der internationale Handel mit Dienstleistungen, der die rückläufigen physischen Warenströme bisher kompensiert und kontinuierlich zugenommen hatte, ist im Rückgang begriffen. Der Indikator globaler ökonomischer Unsicherheit erreichte bereits im August 2019 den höchsten Wert seit Beginn seiner Messung. ${ }^{2}$ Dies reflektierte vor allem die Zurückhaltung bei ausländischen Investitionen und schlug über den hohen Importanteil von Kapitalgütern auf globale Wertschöpfungsketten durch. Tatsächlich verweisen die beiden vorangegangenen großen Schocks der jüngeren Wirtschaftsgeschichte - Weltfinanzkrise, Tohoku-Erbeben (Fukushima) - darauf, dass die Fähigkeit der Weltwirtschaft begrenzt ist, derartige Entwicklungen zu puffern. Wirtschaftliche Erholungsprozesse greifen nach der Krise oft mit erheblicher Verzögerung und verlaufen dann regional ebenso wie zeitlich divergent.

Da die Zunahme der globalen Wirtschaftsverflechtung einhergegangen ist mit einer verstärkten Regulierung ${ }^{3}$ und weil zudem geoökonomische Überlegungen an Raum gewinnen, ${ }^{4}$ wird das Gestalten - möglicherweise das Dominieren - globaler Wertschöpfungsketten immer wichtiger. Der seit mehreren Jahren schwelende Streit zwischen den USA und China macht dies sichtbar. Die Welt droht, in Bezug auf die Lieferverflechtungen in zwei Teile zu zerfallen. Der Freihandel wird zunehmend durch einen Zwang zur Kontrolle von Wertschöpfungsketten ersetzt. Damit könnte der Globalisierungsschub, der mit der Öffnung Chinas im Jahr 1978 bzw. dem Fall des Eisernen Vorhangs im Jahr 1989 begann, und bei den oben genannten Schocks bereits ins Stottern geriet, zum Stillstand kommen. Es entsteht eine Situation verteilter ökonomischer Interessenssphären wie zu kolonialen Zeiten. Deutlich wird dies am von China inzwischen propagierten Konzept der zwei Kreisläufe - einem auf Autarkie ausgerichteten internen Kreislauf, bei dem ausländische Unternehmen allenfalls als Technologielieferanten, solang dies (noch) erforderlich ist,

2 World Trade Organization (WTO): Services trade growth weakens as COVID-19 crisis hits global economy, WTO Website, 11. März 2020; https://www.wto.org/english/news_e/news20_e/wtoi_11mar20_ e.pdf, s. a. World Trade Organization (WTO): Trade Statistics and Outlook. WTO lowers trade forecast as tensions unsettle global economy. WTO Press Release 1.Oktober 2019; https://www.wto.org/english/ news_e/pres19_e/pr840_e.htm. Der Global economic policy uncertainty-Indikator der WTO wird aus der Häufigkeit abgeleitet, mit der Aussagen zu monetären und fiskalischen Sachverhalten und Handelspolitik in Pressemitteilungen mit Unsicherheit verbunden werden. 3 Dafür typisch sind die Compliance-Forderungen an Umwelt und Arbeitsbedingungen, welche die Kontrolle der Lieferketten erzwingen. 4 Diese laufen darauf hinaus, Rohstoffe oder Technologien im Sinne einer strategischen Industrie- und Handelspolitik zum nationalen Vorteil auszuschöpfen. 


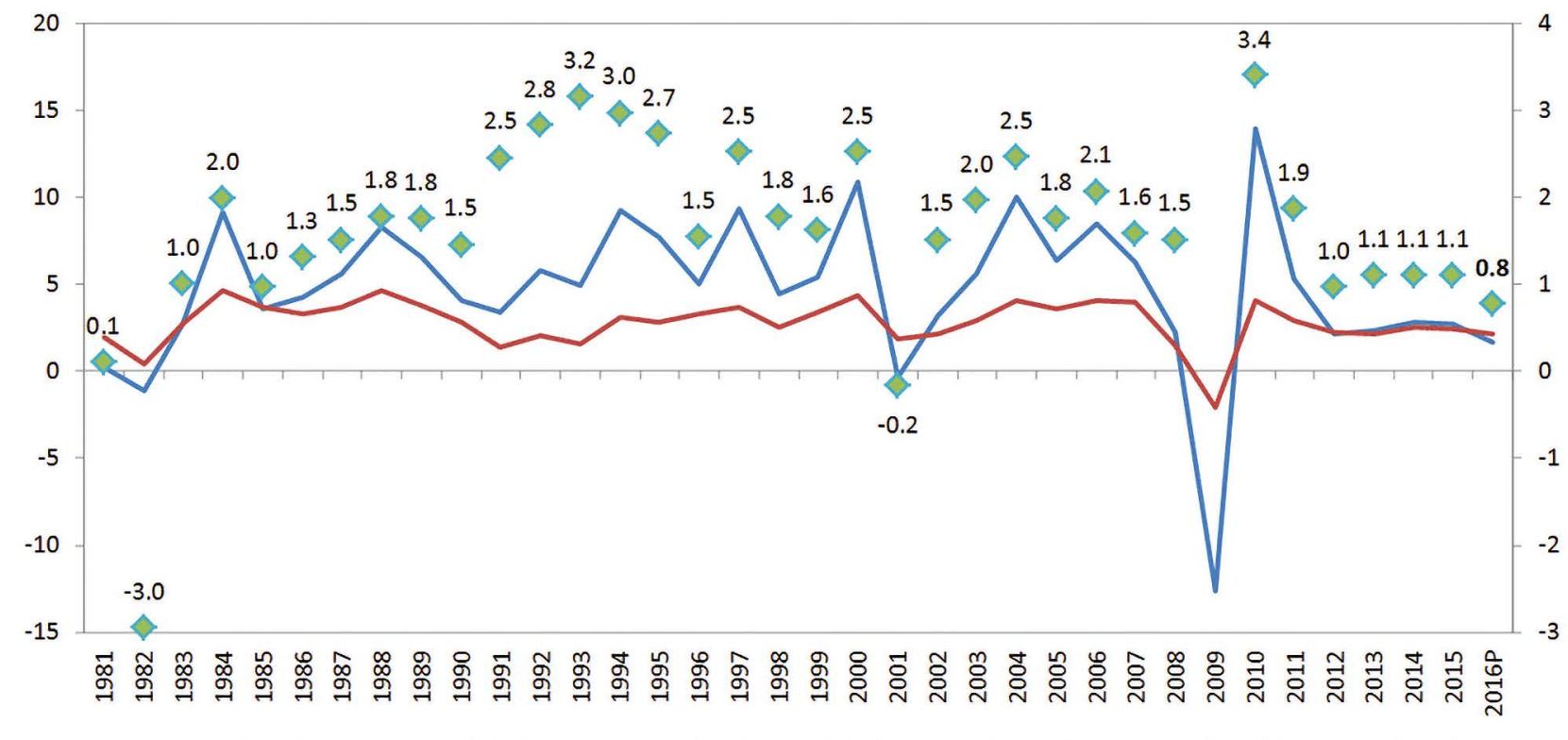

World trade volume growth (left) —World GDP growth (left) Ratio of trade growth to GDP growth (right)

Sources: WTO Secretariat for trade, concensus estimates for GDP.

Abb. 2: Entwicklung der Globalisierung, 1981 bis 2016

Quelle: World Trade Organization (WTO): Trade in 2016 to grow at slowest pace since the financial crisis. WTO Press Release, 27.09.2016; https://www.wto.org/english/news_e/pres16_e/pr779_e.htm. Erläuterung: Ein Wert des Quotienten aus Welthandel und Weltproduktion (grüne Raute) von 1,0 bedeutet ein gleich starkes, ein Wert >1,0 ein überdurchschnittliches und ein Wert $<1,0$ ein unterdurchschnittliches Wachstum des Welthandels im Vergleich zur Weltproduktion.

auftreten, und einem externen, der aber so strukturiert sein soll, dass sich keine strategischen Abhängigkeiten ergeben.

Die im Jahr 2015 erstmalig vorgestellte Strategie Made in China 2025, die auf dem Parteitag der KPC im Oktober 2017 verabschiedet wurde, kann als Versuch gewertet werden, strategische Wirtschaftspolitik mit dem Ziel einer Änderung der globalen Wohlstandsverteilung durchzuführen. Sie findet ihren wohlstandstheoretischen Hintergrund in Analysen, welche die Verteilung der Wertschöpfung entlang von Lieferketten oder -netzwerken beschreiben und nachweisen, in welchem Umfang die globale Arbeitsteilung bisher den Westen begünstigt hat. Abbildung 3 verdeutlicht dies beispielhaft durch die Zuordnung von Wertschöpfungspositionen zu einzelnen Ländern, dargestellt mittels ihrer Landesflaggen. Die Darstellung zeigt, dass es die frühen und die späten Stufen der Wertschöpfungsketten sind, die Länder reich machen - zu Lasten der sogenannten Werkbänke der globalen Wirtschaft - und dass damit ein Verteilungsproblem zwischen den Schwellenländern und den frühindustrialisierten Ländern entsteht. ${ }^{5}$

5 Vgl. hierzu Escaith/Inomata 2016 sowie Inomata 2017.
Die zeitliche Dynamik belegt, dass diese „Lachkurve“ weiter nach unten durchhängt - die rein industriellen Prozesse verlieren an Gewicht. ${ }^{6}$ Unter den Bedingungen des Pariser Klimavertrags und damit der Notwendigkeit, Kreislauffähigkeit von Wertschöpfungsketten zu erhöhen, könnten sich weitere hochwertige Wertschöpfungspotentiale ergeben, die insbesondere Knappheit bei Rohstoffen auslösen. Das bedeutet, dass die später angesprochenen Seltenen Erden an Gewicht für die Wirtschaftspolitik gewinnen, die Technologien und Wertschöpfungspotentiale nach China ziehen soll.

Tatsächlich zeigt China deutlich, dass vor allem Humankapitalstrategien, die den Aufbau einer eigenen Hochtechnologie ermöglichen, sehr erfolgreich sein können. Dabei geht es darum, bisher nicht vorhandene Produktionsmöglichkeiten nach und nach bereitzustellen. In diesem Zusammenhang ist insbesondere die Wirkung der Hochschulreform zur Jahrtausendwende zu nennen. ${ }^{7}$ Dies ermöglichte eine teilweise Entkopplung von internationalen Wertschöpfungsketten, indem Vorleistungen durch eigene Produktion substituiert wurden. Abbildung 4

6 World Intellectual Property Organization 2017.

7 Pei 2018 (Vortrag). 


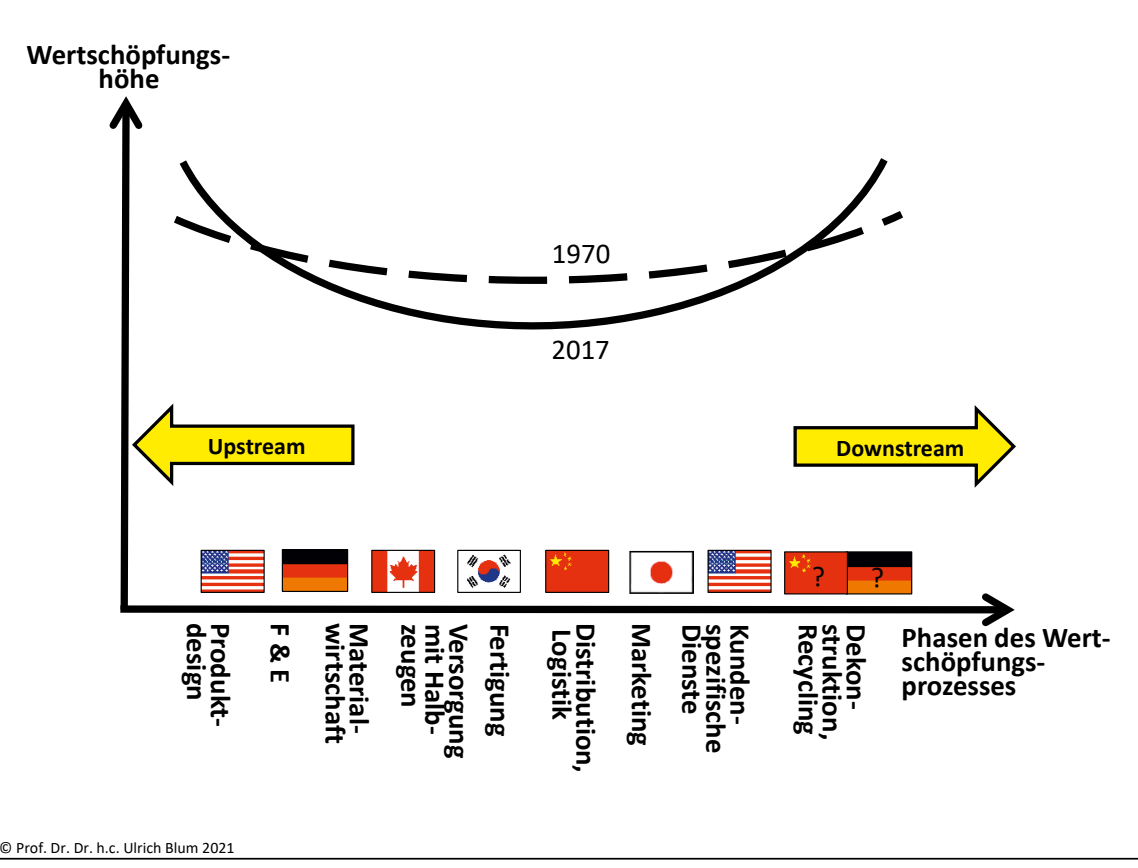

Abb. 3: Positionierung ausgewählter Länder in der globalen Lieferverflechtung Quelle: Blum 2020

belegt, dass in den vergangenen 20 Jahren die importierten Vorleistungen vieler Industrieländer gestiegen und die relativen Eigenproduktionsanteile im Gegenzug gesunken sind. Nur in China ist eine gegenläufige relative Deglobalisierung zu beobachten, die sich jedoch angesichts der zunehmenden Weltmarktrelevanz Chinas zukünftig in weiteren Industrieländern fortsetzen könnte.

Damit ergibt sich ein historischer Anknüpfungspunkt mit der Analyse des deutschen Reichsbankpräsidenten Hjalmar Schacht. Dieser hatte 1937 ausgeführt, dass der Freihandel zwar das beste Wettbewerbsregime der Welt sei, sobald aber Ressourcenknappheiten von wirtschaftlichen Rivalen strategisch eingesetzt werden würden, um sich Wertschöpfungspotentiale zu sichern, würde Kolonialismus zu einer zweckmäßigen Strategie, um die Überlebensfähigkeit einer Nation zu gewährleisten. ${ }^{8}$

Ziel des Beitrags ist es $\mathrm{zu}$ untersuchen, welcher Zusammenhang zwischen der Vulnerabilität von globalen Wertschöpfungsketten, der zugrundliegenden Kritikalität (vor allem bezüglich Rohstoffstrategien) und dem Raum, genauer den Markt-Wettbewerbskonstellationen besteht. Es wird zudem analysiert, welche Abwehr- und Immunisierungsstrategien ergriffen werden können, d.h. wie

8 Hjalmar Schacht warnte vor den Folgen dieser Zerstörung des Freihandels - damals als Folge des Kolonialismus - für die Sicherheit; seine Analyse gilt heute als Menetekel der Folgen einer Zerstörung der marktwirtschaftlichen Weltwirtschaftsordnung, Schacht 1937.
Unternehmen und Länder, die auf wenige Branchen spezialisiert sind und deren Risikoprofil teilen, Vulnerabilität reduzieren können. Dies hat hohe Relevanz angesichts der sicherheitspolitischen Dimension Seltener Erden, die hier beispielhaft herangezogen werden, und der aus Gründen der globalen Nachhaltigkeit forcierten weltweiten Transformation zu einer Circular Economy. Es werden strategische Abhängigkeiten reduziert, auch wenn damit möglicherweise Arbitragegewinne der Globalisierung verloren gehen, wie dies im Kontext der klassischen Handelstheorie üblicherweise unterstellt wird. ${ }^{9}$

Der Beitrag ist wie folgt gegliedert: Im zweiten Abschnitt wird ein Vorschlag zur Messung von Vulnerabilität vorgetragen. Der dritte Abschnitt widmet sich einer Analyse der strategischen Vulnerabilität unterschiedlicher Markt-Wettbewerbskonstellationen und eruiert Abwehrund Gestaltungsmaßnahmen für eine Governance von Vulnerabilität. Gesucht wird die Antwort auf die Frage, wie eine Governance der Vulnerabilität auf Ebene des ein-

9 Allerdings zeigt die Neue Handelstheorie, dass durch gezielten Aufbau positiver Externalitäten, also vereinfacht gesagt durch das Überspringen der Erträge aus Innovation auf Dritte, die keine hierauf bezogenen Investitionen getätigt haben, z. B. begünstigt durch den Veröffentlichungszwang in Patentschriften, auch das Gegenteil möglich ist (Blum 2018); zugleich kann auch durch gezielte Verkehrsinvestitionen die Handelsstruktur nachhaltig zum eigenen Vorteil verändert werden, wie dies die Seidenstraßeninitiative der chinesischen Regierung belegt (Blum 2019). 


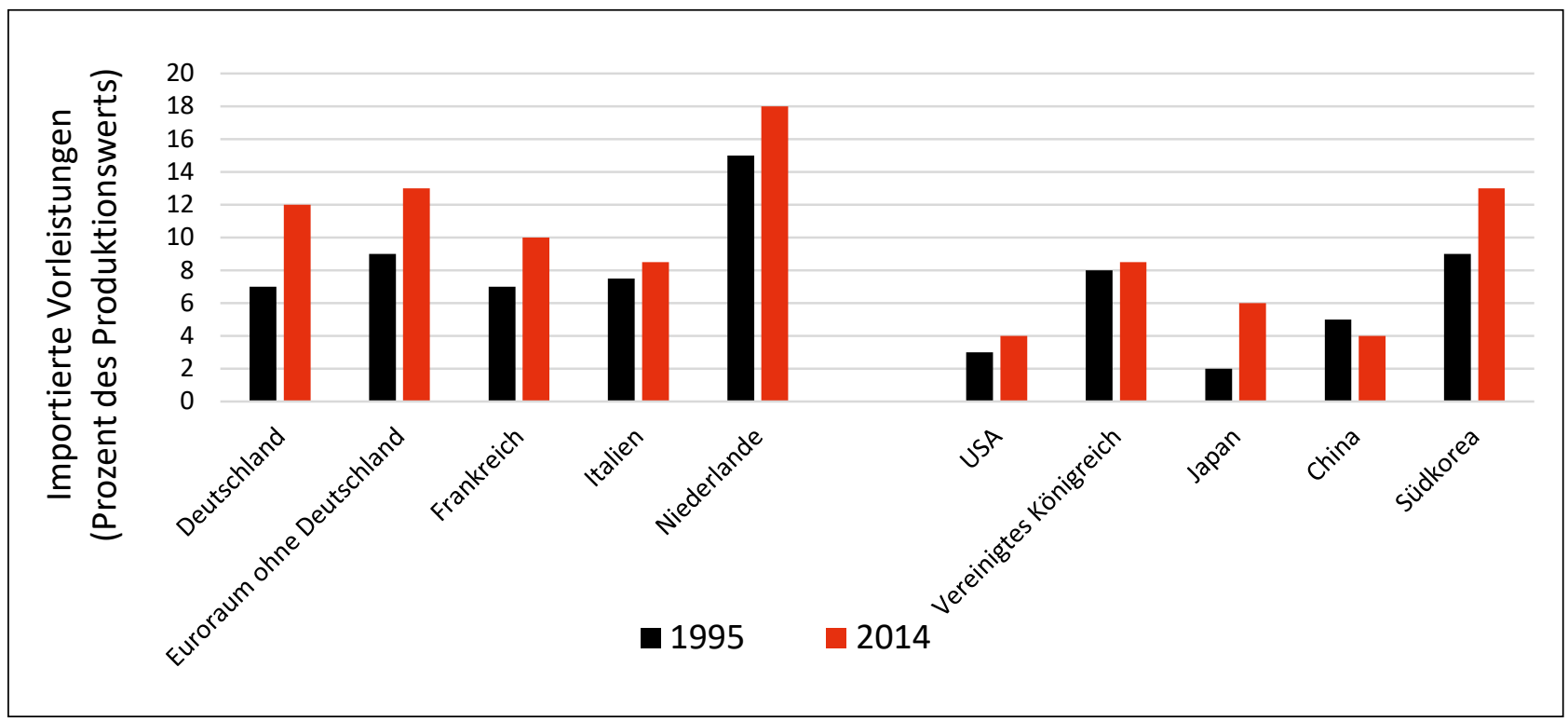

Abb. 4: Entwicklung der gesamtwirtschaftlichen Importanteile Quelle: eigene Darstellung nach Deutsche Bundesbank 2018

zelnen Unternehmens bzw. wertschöpfungskettenübergreifend umgesetzt werden kann.

Im vierten Abschnitt werden konkrete Rohstoffstrategien anhand von Hochleistungsmagneten und dem zugrunde liegenden Materialstrom der Seltenen Erden exemplarisch diskutiert. Es soll dabei die strategische Vulnerabilität der gesamten Wertschöpfungskette im Vordergrund stehen anstelle einer partikulären Sicht auf Branchenrisiken. Die große Zahl an Anwendungen Seltener Erden macht deren Wertschöpfungsketten undurchsichtig, deshalb sind hier die Fähigkeit zur Kodifizierung, d.h. zur Normung, aber auch der Wille zur Kodifizierung prägend für die Industriestruktur. ${ }^{10} \mathrm{Im}$ abschließenden fünften Abschnitt folgen eine kritische Analyse der zunehmend geforderten Rohstoff- und Technologiesouveränität und Vorschläge zu Politikmaßnahmen.

\section{Kritikalität, Risiko und Vulnerabilität}

\subsection{Vulnerabilität globaler Wertschöpfungs- ketten}

Vulnerabilität wird in seiner globalen Wertschöpfungsketten-Dimension durch bestehende Modelle unzureichend

10 Machacek/Fold 2018 mit Bezug auf Gereffi 2011. erfasst. Diese messen üblicherweise vergangenheitsbezogene Schwankungen der Aktienrendite. Sie sollten jedoch eher das Ertragsrisiko erfassen, beispielsweise als Variationskoeffizient der Gewinne, welches über Investition und Beschäftigung unmittelbare Wirkungen auf die Volkswirtschaft als Ganzes ausübt. ${ }^{11}$ Unternehmen und Volkswirtschaften sind verletzlich, also vulnerabel, wenn ihnen bestimmte Rohstoffe oder infolge von deren Verknappung bzw. technologischen Engpässen Komponenten und Teile fehlen, die nicht kurzfristig zu substituieren sind. Bezogen auf Verflechtungsanalysen sind damit Rohstoffe, Teile oder auch solche Technologien als kritisch anzusehen, die erhebliche ökonomische Hebelwirkungen verursachen - sogenannte Anstoßeffekte. ${ }^{12}$ Der Begriff der Kritikalität besitzt folglich zwei Dimensionen: Verfügbarkeit und Vulnerabilität. Das können die Folgen von Preisspitzen sein, aber auch einer Verknappung und möglicherweise auch einer Nichtverfügbarkeit. ${ }^{13}$ Heute kommen noch die Umweltfolgen der bergmännischen Extraktion,

11 Gleißner 2018, 56 u. [63], Fn. 47.

12 Vidal et al. 2013 ordnen diese Kritikalität auch „konventionellen“ Rohstoffen wie Bauxit (Aluminium) oder sogar Zement im Kontext einer forcierten Energiewende zu; für die Bauwirtschaft vieler Länder existiert bereits eine erhebliche Kritikalität geeigneter Sande.

13 Wäre Kupfer kritisch, dann ließe es sich bei leitungsgebundener Netzinfrastruktur durch Glasfaserleitungen ersetzen (materielles Äquivalent). Man könnte aber auch das Gesamtsystem durch eine drahtlose Infrastruktur substituieren (funktionales Äquivalent). Die Kommunikation ließe sich sogar durch eine persönliche Reise mit dem Pkw zum Gesprächspartner leisten (Nutzenäquivalent). 
der Anreicherung, Verarbeitung und Entsorgung der Endprodukte am Ende des Lebenszyklus hinzu. Ein Rohstoff kann auch dadurch kritisch werden, dass er am Ende des Lebenszyklus nicht angemessen aufbereitet bzw. entsorgt werden kann. Maßstab ist dann die endliche Deponiekapazität der Erde. Eigentlich sollten sich die drei Dimensionen (Verfügbarkeit, Vulnerabilität, Umweltfolgen) in den (Knappheits-) Preisen der Rohstoffe widerspiegeln - sie tun dies aber nur begrenzt.

Vulnerabilität kann für Firmen bedrohlich werden, weil viele Unternehmen ähnliche Produkte herstellen oder Funktionen auf sehr unterschiedlicher Werkstoffund damit Rohstoffbasis erfüllen: Wenn beispielsweise Neodym und Dysprosium knapp werden, dann sind die Hersteller der entsprechenden Magnete unter Druck, während solche auf Basis von Samarium und Kobalt weniger betroffen sind. Gehen Erstere in Insolvenz und trifft der nächste Preiszyklus Kobalt, dann werden auch diese ausscheiden und allein Elektromagnettechnologien überleben. Aus diesem Grund ist das Risikomanagement eines Unternehmens eine wesentliche Basis, um entsprechende Probleme zu vermeiden, vorrangig in der Abfolge der Risikoidentifikation, Risikobeurteilung und Risikosteuerung. ${ }^{14}$

Ursprünglich wurde das Konzept der Vulnerabilität im ökologischen Kontext auf sozio-ökonomisch und geographisch bedingte Risiken, beispielsweise klimawandelinduzierte Nahrungsmittelunsicherheit und Katastrophen, angewendet. ${ }^{15}$ Heute trifft es die Breite der durch kritische Ver- und Entsorgungslagen gegebenen ökonomischen Engpässe und findet sich auch in verwandten Diskussionssträngen zur Resilienz bzw. Robustheit lokaler Wirtschaftskreisläufe. ${ }^{16}$

Für komplexe Wertschöpfungsketten in Maschinenbau und Elektronik gilt, dass sowohl der Güter- und Informationsstrom als auch die zugrundeliegende Infrastruktur zunehmend long and lean gestaltet sind.${ }^{17}$ Deshalb gibt es dort einen hohen Grad an Abhängigkeit von Lieferanten, so dass Risiken (auch informationelle) in langen, d. h. über viele Zwischenstufen reichenden Wertschöpfungsketten, weitergereicht werden. Angebots- oder Nachfrageschocks „fressen“ sich durch die Lieferverflechtungen und werden kaum abgepuffert. So hat die globale Finanzkrise 2009 zunächst die Nachfrage innerhalb eines Halbjahreszeitraums international um 20-30 Prozent reduziert und die Preise auf allen Wertschöpfungsstufen um bis zu

14 Gleißner 2001.

15 Adger 2006.

16 Quoreshi/Stone 2019.

17 Peck 2005, 215. Svensson 2000, 732.
40 Prozent zurückgehen lassen mit der Folge einer hohen Zahl von Lieferanten-Insolvenzen. In den USA trug das zu einer Phase nachhaltig gebremsten Wachstums bei. ${ }^{18}$

Die im Supply Chain Management am häufigsten auf Wertschöpfungsketten-Risiken untersuchten Einzelbranchen sind mit der Automobilindustrie und der Luftfahrtindustrie solche, die sich stark am Endprodukt orientieren und von global tätigen Original Equipment Manufacturers (OEMs) dominiert sind. Sie weisen zugleich eine hohe Materialkomplexität (beispielsweise leichte Verbundwerkstoffe) sowie eine hohe Kontraktintensität auf. Sie sind also durch eine Vielzahl von Lieferanten entlang der Lieferkette - meist aus unterschiedlichen Ländern gekennzeichnet. ${ }^{19}$ Dies erlaubt es, Verwundbarkeiten zu vermeiden oder in ihren Auswirkungen klein zu halten. So kann etwa durch branchenübergreifende Lieferantendiversifikation das Risiko gesenkt werden und bei der Produktion von Hochleistungselektronik in China besteht eine Komplementarität, keine Rivalität mit ostasiatischen Ländern wie Japan, Südkorea und Taiwan. ${ }^{20}$ Durch Lieferantendiversifikation lassen sich allerdings nicht alle Verwundbarkeiten beseitigen. Die hohe Fragmentierung der pharmazeutischen Wertschöpfungskette hinsichtlich beteiligter Lieferanten und Länder hat den Missbrauch paralleler Vertriebskanäle (pharmaceutical diversion) zur Produktfälschung begünstigt. ${ }^{21}$ Zudem entstehen aufgrund der uneinheitlichen Branchenstandards und Legislativen zusätzliche Risiken.

Ein wesentlicher Auslöser von Vulnerabilität ist die Kritikalität solcher Ressourcen, ohne die bestimmte, wesentliche Güter nicht oder nicht effizient hergestellt werden können. Anfang des Jahrzehnts rückten Seltene Erden ins Zentrum der Analyse, weil infolge eines geostrategischen Streits zunächst mit Japan China die globale Belieferung massiv verknappte (Abbildung 5). Die für nahezu alle technologischen Anwendungen vom Automobil bis zu Kleinelektrogeräten benötigten Hochleistungsmagnete beruhen auf Seltenen Erden. Sie sind daher Teil einer Hochrisiko-Wertschöpfungskette, was sich auch im Zuge der durch China verhängten und durch die WTO im Jahre 2014 sanktionierten Exportbeschränkung für Seltene

18 Jüttner/Maklan 2011, 254.

19 Dollar/Kidder 2017, $161 \mathrm{f}$. Kontraktintensität steigt mit der Zahl der beteiligten Lieferanten, vergleichbar der Länge und Fragmentierung der WK, und mit dem Anteil an entwickelter Technologie (162). Ein illustrierendes Beispiel sind Mobiltelefone, die als kontraktintensive Technologie viele proprietäre Eigentumsrechte bündeln, sichtbar an der hohen Zahl an Patentkriegen in den Branchen Telekommunikation und Elektronik.

20 Gereffi 2011.

21 Enyinda/Szmerekovsky 2008. 


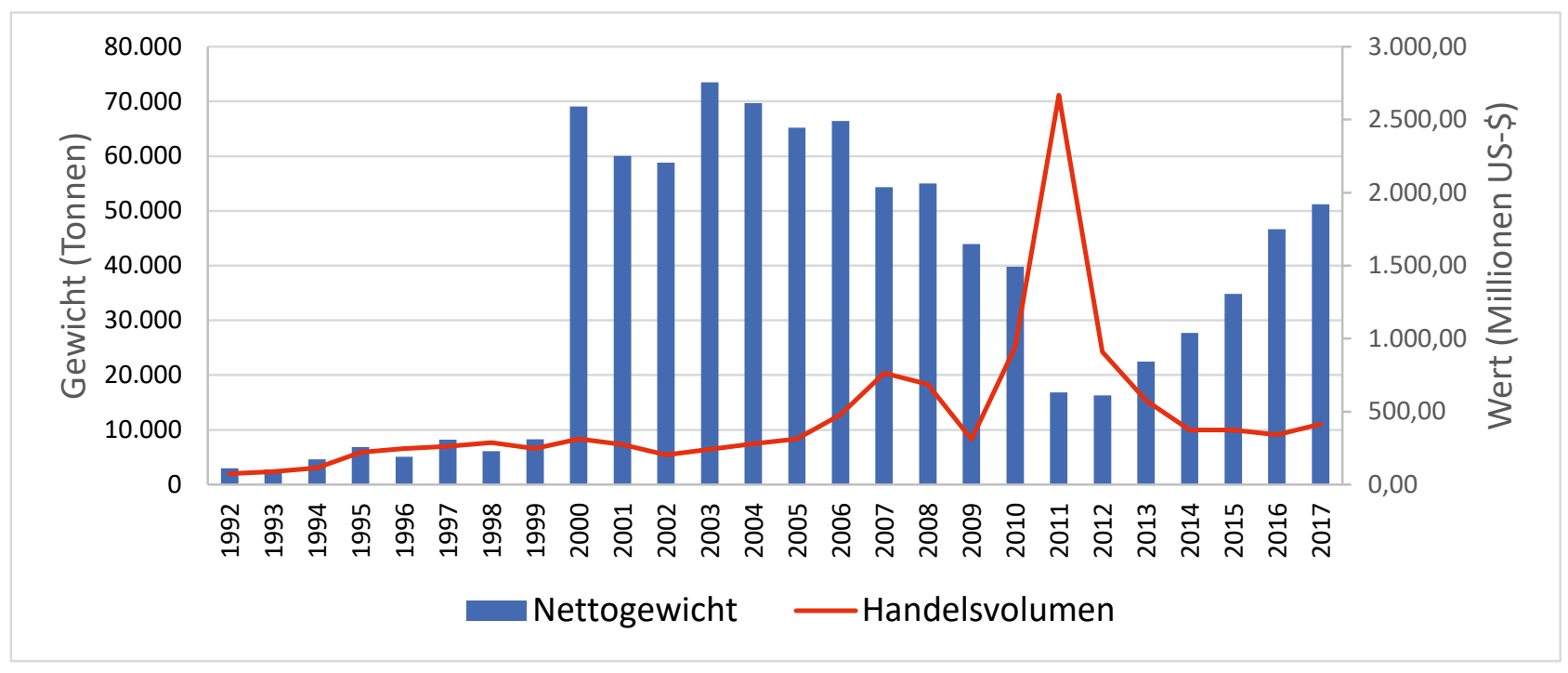

Abb. 5: Wert- und Mengenentwicklung bei Seltenen Erden, 1992 bis 2017 Quelle: eigene Darstellung nach UNCOMTRADE.

Erden gezeigt hat. ${ }^{22}$ Die chinesischen Exportbeschränkungen sind vornehmlich außen- und machtpolitisch motiviert und reflektieren den Anspruch Chinas, eine globale Welt- und Führungsmacht zu sein.

In diesem Zusammenhang wird der Zugang $\mathrm{zu}$ Wertschöpfungsketten zum Kristallisationspunkt wirtschafts- und sicherheitspolitischer Strategien. So haben amerikanische Zölle auf chinesische Güter von ,industrietechnologischer Bedeutung“ bereits eine Neustrukturierung von Wertschöpfungsketten zur Folge gehabt. ${ }^{23}$ Dies wurde sichtbar an der Teilverlagerung des Technologieunternehmens Foxconn von China nach Amerika und dem weitgehenden Rückzug von Huawei aus dem amerikanischen Forschungsstandort. Nicht nur bei Hightech- und Greentech-Produkten, sondern auch bei der Militärtechnologie (von Generatoren in Jet-Motoren bis zu Navigations-

22 Als besonders versorgungskritisch gilt das schwere Selten-Erdelement Dysprosium, ein zentraler Bestandteil von Hochleistungsmagneten für Windräder und Elektromobilität, vgl. Glöser-Chahoud/Tercero Espinoza 2015, 9. Risikobehaftet sind auch die mit Gewinnung und Verarbeitung verbundenen Umweltlasten wie die Koextraktion radioaktiver Elemente, Emissionen und hoher Energieverbrauch, die in Lebenszyklusanalysen (life cycle assessments) erfasst werden können, vgl. z. B. Pell et al. (2019), 65.

23 Über 90 Prozent der im Juli 2018 in Kraft gesetzten amerikanischen Zölle in Höhe von 25 Prozent auf chinesische Importgüter entfallen auf Zwischenprodukte und Investitionsgüter, darunter Motoren, Landwirtschafts- und Industriemaschinen sowie Test-, Messund Diagnose-Instrumente, ein geringer Teil nur auf Konsumgüter, vgl. Martin Lanz: Der Schlagabtausch zwischen den USA und China nimmt seinen Lauf, Neue Zürcher Zeitung, 06.07.2018. systemen von Präzisionslenkwaffen) ist die amerikanische Volkswirtschaft abhängig von Seltenen Erden aus China. ${ }^{24}$ Bei hochwertigen Mikrochips besteht eine Abhängigkeit von Taiwan. Dieser Umstand wird natürlich sicherheitspolitisch und strategisch durch China genutzt und ist Teil des amerikanisch-chinesischen „Wirtschaftskriegs“. ${ }^{25}$

Bestimmte Eigenschaften machen Wertschöpfungsketten besonders verwundbar. Hierzu zählt die Abhängigkeit von wenigen Lieferanten, im Extremfall von nur einem (single sourcing), oder von nur wenigen Kunden. Beides kann externe Schocks verstärken. Wenn derartige disruptive Risiken upstream oder downstream auftreten und infolge des Überschreitens einer tolerierbaren Schwelle Vulnerabilität begründen, ${ }^{26}$ dann können sich über die Weitergabe entlang oder in Gegenrichtung der Wertschöpfung Risikofolgen verstärken. ${ }^{27}$ Damit gewinnen drei Begriffe für die Messung von Vulnerabilität an Bedeutung, nämlich die Exposition, wie stark also eine

24 Carpenter 2012, 416. Wie er weiter ausführt, sind Seltene Erden im Unterschied zu anderen kritischen Metallen nicht in die Berry Amendment's Specialty Metal Provision einbezogen, die Lieferanten verpflichtet, bei allen Verträgen mit dem US-Verteidigungsministerium 100 Prozent der in Rüstungsgütern verarbeiteten Metalle innerhalb der Vereinigten Staaten zu beschaffen (427).

25 Blum 2020.

26 Bugert/Lasch 2018 sprechen dann im Anschluss an Heckmann et al. 2015 von supply chain disruption risk.

27 Wagner/Bode 2006, 308. Wie die Autoren anmerken, wurden zwar (hoch-)signifikante Zusammenhänge gefunden, aber die erklärte Varianz ist in allen Modellen gering, was nahelegt, dass die Vulnerabilität erklärenden Variablen unvollständig sind (308f.). 
Wertschöpfungskette Risiko ausgesetzt ist, zweitens die Sensitivität, also die Stärke der Reaktion auf Schocks, und drittens die Anpassungskapazität, also die Fähigkeit, Gegenmaßnahmen zu ergreifen. ${ }^{28}$

\subsection{Indikatoren der Vulnerabilität}

Abbildung 6 zeigt die Anforderungen an die Messung von Vulnerabilität entlang der drei Dimensionen, nämlich die Exposition gegenüber Schocks in der Wertschöpfungskette, ihre Reaktion auf diese Schocks, also die Sensitivität, und schließlich Anpassungskapazität. Der Begriff „Raum“ ist hier übergreifend gemeint, erfasst also den geographischen Raum, aber auch Produkt- und Technologieräume oder Zeiträume. So erhöht eine hohe Bestreitbarkeit des Markts, also eine hohe Wettbewerbsintensität, die Wahrscheinlichkeit von Schocks. Die Einbettung der Wertschöpfungskette im Raum, insbesondere die Stärke der Kopplung zwischen vor- und nachgelagerten Stufen sowohl geographisch wie produktspezifisch, und die interaktive Komplexität (das Ausmaß an gegenseitiger Abhängigkeit von Inputs in der Produktion), erhöhen ebenfalls die Vulnerabilität. Hoch ist die interaktive Komplexität beispielsweise bei chemischen Prozessen und in der Stahlproduktion, wie Wagner/Neshat 2012 ausführen. Die Kapazität der Puffer, die also Wirkungen im „Zeitraum“ abschwächen können, spielt ebenso eine Rolle. ${ }^{29}$

Hier werden einige Indikatoren herausgegriffen, die die drei Dimensionen der Exposition, Sensitivität und Anpassungskapazität beeinflussen und die den zu Beginn erwähnten Bezug zum Markt sowie zu materialintensiven Wertschöpfungsketten herstellen, was selten Teil der Vulnerabilitätsanalyse ist.

Bei der Exposition ist die Bestreitbarkeit des Marktes (contestability) zentral, diese ist ein Maß für die Anfälligkeit der eigenen Marktposition gegenüber Konkurrenten und damit ein Faktor, der Vulnerabilität erhöht. ${ }^{30}$ Als normatives Ideal von Märkten ist Bestreitbarkeit dann gegeben, wenn Markteintritt und -austritt und die dabei auftretende Verlagerung von Vermögenswerten ohne

28 Diese Einteilung geht auf die frühesten Vulnerabilitätsanalysen zu Klimarisiken zurück, die bei Adger 2006 referiert werden.

29 In Gesprächen mit Banken wurde den Verfassern des Artikels regelmäßig bedeutet, dass sich Lagerkapazitäten in der Nach-CoronaZeit verdoppeln werden, um über Puffer zu verfügen.

30 Dies gilt in jedem Fall auf der Unternehmensebene, dürfte aber auch für Cluster, integrierte Wertschöpfungsketten oder Länder Relevanz besitzen, auch wenn das Konkurrenzkonzept von Unternehmen nicht nahtlos auf Cluster oder Länder übertragen werden kann; vgl. Krugman 1994.
Verlust (ohne versunkene Kosten) möglich sind und somit auch keine Ein- und Austrittsbarrieren im Markt bestehen. ${ }^{31}$ Treffen externe Schocks auf Marktstrukturen mit hoher monopolitischer Konkurrenz, ergeben sich sehr unterschiedliche Fähigkeiten und Formen des Abpufferns. Wenn Marktmacht auf bestimmten Kritikalitäten aufbaut, also vor allem Rohstoffen und Verfahren, kann ein bis dahin stabiles System zusammenbrechen, ohne dass die Dynamik des Marktes die Kritikalität wieder beseitigt. Die eigentlich starke Marktstellung des Monopols ist also potentiell insofern vulnerabel, als sie durch technologisch disruptive Wettbewerber mit besserem Ressourcenzugang oder innovativen Verfahren bedroht werden kann. Auch unterlassene Investitionen im Bereich Forschung und Entwicklung können die Position von Monopolisten unterminieren. Es wurde jedoch gezeigt, dass es Monopolisten unter den Bedingungen unvorhersehbarer Preissetzung bei komplexen Produkten wie Smartphones oder durch überraschende Preissenkungen durch Großhandelsunternehmen möglich ist, Konkurrenten vom Markteintritt abzuhalten und eine Monopolrente zu erwirtschaften. ${ }^{32}$ Das spricht gegen eine vollständige Bestreitbarkeit, auch wenn keine Irreversibilität vorliegt. Der Monopolist kann sich gegen Vulnerabilität durch Wettbewerber unter diesen Umständen immunisieren.

In materialbasierten Wertschöpfungsketten erhöht die Kritikalität von strategischen Ressourcen, beispielsweise von Technologiemetallen, die Sensitivität und damit die Vulnerabilität, auch wenn diese nur in geringer Menge für die Herstellung von Hightech-Produkten benötigt werden. ${ }^{33}$ Zwar wird eine Fehldeckung zwischen Angebot und Nachfrage, also die klassische Knappheit, als wichtiger Treiber von Wertschöpfungsketten-Vulnerabilität häufig genannt, jedoch meist mit Bezug auf den downstream-Abschnitt der Wertschöpfungskette, d.h. die Zwischenprodukte und Endprodukte. ${ }^{34}$ Dagegen wirkt sich auch upstream eine Fehldeckung zwischen Angebot und Nachfrage disruptiv aus, beispielsweise ein Nachfrageüberhang nach schweren Seltenen Erden, da deren anteiliges Vorkommen in der Erdkruste nicht dem Verhältnis entspricht, zu dem sie durch Erz- und Magnetproduzenten nachgefragt werden. ${ }^{35}$ Kritikalität, v.a. bezüglich der schweren Seltenen Erden wie Dysprosium, setzt die Wertschöpfungskette für Hochleistungsmagnete an ihrem upstream-Ende einer hohen Vulnerabilität aus, denn es

\footnotetext{
31 Baumol/Lee 1991, $2 \mathrm{f}$.

32 Braido/Shalders 2015.

33 Blum/Schmid 2015.

34 Wagner/Neshat 2012.

35 Glöser-Chahoud/Tercero Espinoza 2015, 9.
} 


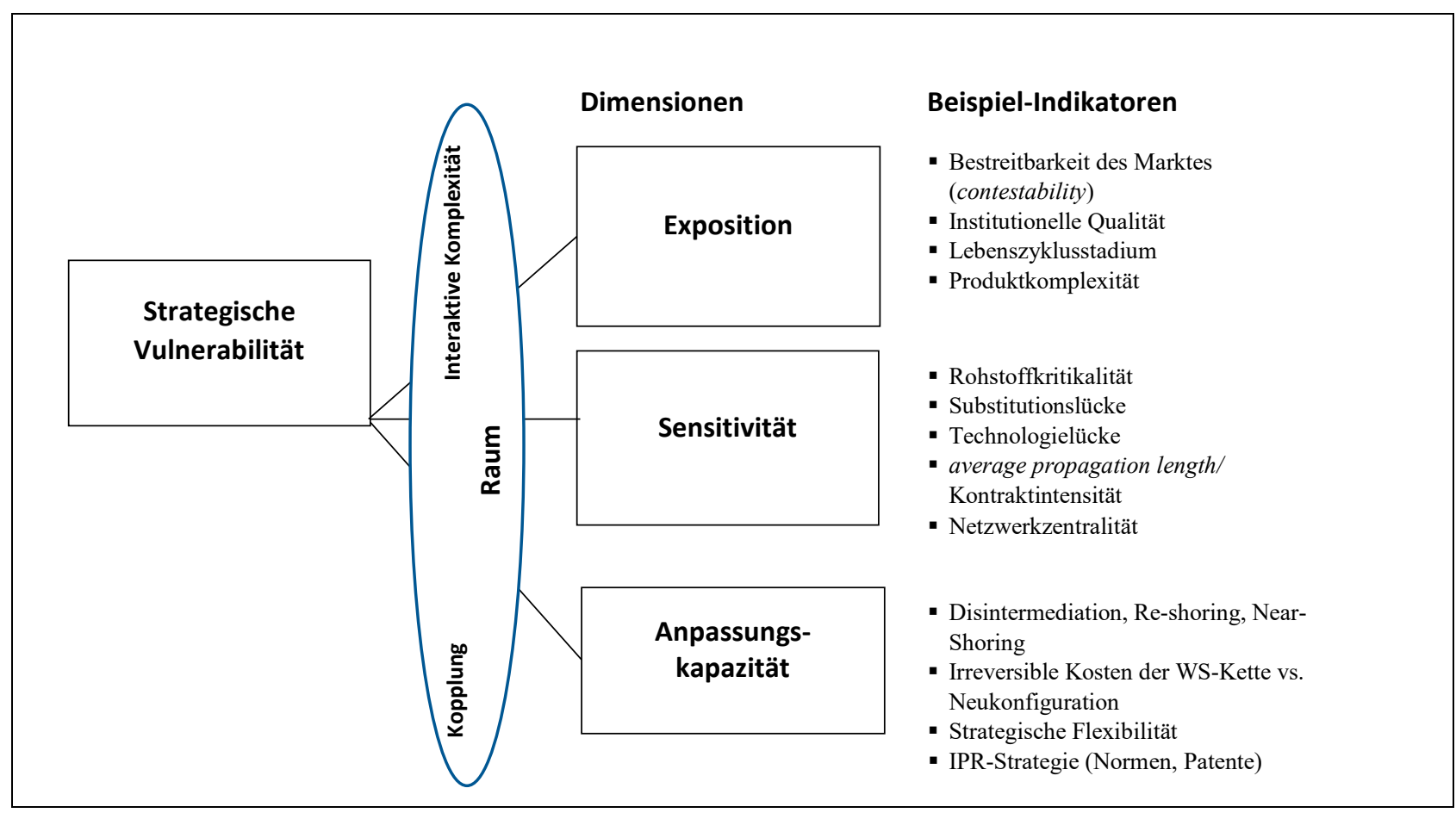

Abb. 6: Indikatoren strategischer Vulnerabilität von Wertschöpfungsketten Quelle: eigene Darstellung und Indikatoren (zu den drei Vulnerabilitätsdimensionen vgl. Adger 2006, zu den Raum-Indikatoren vgl. Wagner/ Neshat 2012)

fehlen alternative Lieferanten, die in ausreichender Menge liefern können. Weitere Sensitivitäts-Indikatoren sind die Technologielücke und Netzwerk-Zentralität. Die Technologielücke als Maß des Entwicklungsrückstands (gemessen beispielsweise an der Produktivitätsdifferenz) zum Wettbewerber, erhöht die Marktaustrittswahrscheinlichkeit. ${ }^{36}$ Ist die Stellung eines Unternehmens in einem Netzwerk zentral mit leichtem Ressourcenzugang und komplementären Verbindungen $\mathrm{zu}$ anderen Unternehmen und Forschungseinrichtungen (hohe Netzwerkzentralität), senkt das die Sensitivität und damit die Vulnerabilität. Dies gilt auch aus der Perspektive der strategischen Position von Ländern. Wertschöpfung ist in zentralen Regionen konzentriert, deren Stellung sich noch dadurch erhöht, dass sie vorrangig in den Handel mit Zwischenprodukten ihrerseits zentraler Regionen eingebunden sind, wobei die regionalen Cluster Nordamerika, Europa und Asien mit den Brückenköpfen China, USA und Deutschland zu nennen sind. ${ }^{37}$

36 Cantner 2007.

37 Escaith 2017, 111. Auch Baldwin 2013 (20 f.) ermittelt sogenannte Headquarter-Economies, zu denen er neben den USA und Deutschland v. a. Japan (noch vor China) zählt, die den größten Teil des Bedarfs an Zwischenprodukten an ressourcenstrategisch abhängige
Die gegenwärtige Verkürzung der Wertschöpfungsketten durch Re-shoring und Near-shoring, durch die größere Teile der Wertschöpfungskette wieder in die Ursprungsländer zurückwandern, dient der Erhöhung der Anpassungskapazität. Anpassungskapazität fußt auf der Qualität des Organisationskapitals. Dieses umfasst das interne, unternehmensspezifische Wissen sowie die externe Einbettung von Unternehmen in Wertschöpfungsbeziehungen. ${ }^{38}$ Durch Kopplung im Raum mit Kooperationspartnern (beispielsweise aufgrund langfristiger Lieferverträge) wird Vulnerabilität ebenfalls erhöht. Die Einbindung von Unternehmen in eine konkrete WertschöpfungskettenKonfiguration besitzt einen hohen Grad an Irreversibilität, kann also nur unter erheblichen Kosten verändert werden. Dabei geht es um die vertikale Arbeitsteilung mit unterschiedlich produktiven, vor- und nachgelagerten Unternehmen. Vertragsbindung an weniger produktive Partner in Wertschöpfungsketten senkt die strategische Flexibilität und damit die Wettbewerbsfähigkeit von Unternehmen, weil ein kurzfristiger Partnerwechsel nicht möglich

Factory-Economies (Canada, Mexiko innerhalb von NAFTA sowie Polen, Spanien, Portugal innerhalb Europas) liefern.

38 Tomer 2008. 
ist. ${ }^{39}$ Auch die industrielle Basis reduziert die Umstellungsbefähigung. So wurde in einer Beispielrechnung der UBS gezeigt, dass es den USA durch die Erhebung eines 25 Prozent-Zolls auf chinesische Smartphones gelänge, bis zu 60 Prozent der Wertschöpfungskette wieder in die USA zurückzuholen. Die Smartphones aus überwiegend amerikanischer Produktion wären dann aber um 17,5 Prozent teurer, ein komplett in den USA gefertigtes Smartphone sogar bis zu 40 Prozent teurer. Eine solche Umstellung der Industriestruktur würde zudem wegen fehlender, meist technologisch verloren gegangener Fähigkeiten, beispielsweise zum Bau der komplexen Smartphone-Kameras, bis $\mathrm{zu} 4$ Jahre dauern und könnte durch ein Ausweichen der Lieferanten auf Länder, für deren Produkte keine Zölle erhoben werden, konterkariert werden. ${ }^{40}$ Vulnerabilität kann weiter durch Strategien zum Schutz bzw. zur Umgehung des Schutzes von Geistigem Eigentum (Intellectual Property Rights - IPR), beispielsweise Patent- und Normungsstrategien, beeinflusst werden, die die Anpassungsfähigkeit erhöhen.

\section{Möglichkeiten der Governance von Vulnerabilität}

Der Umgang mit Kritikalität und Vulnerabilität erfordert von Unternehmen und Staaten ein effizientes Handeln und im Sinne der modernen Governance-Forschung sind derartige Regeln und Routinen ebenfalls knappe, also kritische Güter. Überträgt man das Konzept von Williamson auf Wertschöpfungsketten, dann ist das Ziel, Ordnung zu schaffen, um Konflikte zu mildern und beiderseitigen Gewinn $\mathrm{zu}$ erzielen. ${ }^{41}$ Risikominimierung in resilienten Lieferketten bewegte sich bisher weitgehend auf der Ebene von Einzelunternehmen, meist desjenigen Unternehmens, das als zentraler Knoten in einem Netzwerk identifiziert wird, sowie der unmittelbaren Zulieferer. ${ }^{42}$ Dies reicht in besonders risikobehafteten Branchen, beispielsweise der chemischen Industrie, der Lebensmittelindustrie, der Automobil- und Luftfahrtindustrie und der

39 Cantner/Savin/Vannuccini 2019.

40 Maier-Borst, Haluka/Lemcke, Anja/Hosp, Gerald: „TrumpPhone“: Was Smartphones „made in USA“ kosten würden, Neue Zürcher Zeitung, 29.11.2018.

41 Williamson (2010: 647) ,... in that governance is the means by which to infuse order, thereby to mitigate conflict and realize mutual gain.“

42 Tewari 2013, $41 \mathrm{f}$. Siehe auch die Fallstudien bei Jüttner/Maklan 2011.
Pharmazeutischen Industrie nicht aus. ${ }^{43}$ Seltener wurde die globale Lieferkette als Ganzes betrachtet. ${ }^{44}$ Für eine Einbeziehung der gesamten Wertschöpfungskette in eine Vulnerabilitätsanalyse spricht, dass Produzenten spezialisierter Komponenten, Technologien und Ausrüstungen durch ihre Marktdominanz (parameter-setting firms) die Wettbewerbsfähigkeit aller nachfolgenden Unternehmen beeinflussen, was vor allem für komplexe Materialien gilt. ${ }^{45}$ Tatsächlich stehen Unternehmen, Branchen oder Ländern verschiedene Strategien zur Verfügung, auf Vulnerabilität $\mathrm{zu}$ reagieren, um ein Marktaustrittsrisiko oder strukturelle volkswirtschaftliche Schäden zu vermeiden, die beispielsweise Wagner und Kollegen als Folgen eines fehlenden übergreifenden Risikomanagements von Wertschöpfungsketten monieren. ${ }^{46}$

Die nachfolgende Matrix führt solche generischen Strategien zur Governance von Vulnerabilität auf, wobei zwischen der Unternehmens-/Branchen-/Länderebene und globalen Wertschöpfungsketten sowie der kurz- und langfristigen Perspektive unterschieden wird (Abbildung 7). Die in den Matrix-Feldern genannten Maßnahmen werden im Folgenden überblicksartig erläutert.

\subsection{Unternehmens-, Branchen- und Länder- ebene: Risk shaping}

Gute Governance im Sinne einer Risikominderung, also einer Risikoanalyse und Risikobeeinflussung, unterscheidet zwischen der ex ante- und ex post-Minderung von Risi-

43 Akamp/Mesterharm/Müller 2010, Tewari 2013, 44, Enyinda/ Szmerekovsky 2008. Für Unternehmen der Chemischen Industrie zeigen z.B. Hazzan/Jones 2004 auf, wie eine Vulnerabilitätsanalyse für typische Materialarten und Transportwege gegenüber Angriffen wie Diebstahl, Sabotage und Umweltkontamination durchgeführt werden und eine Risikoteilung zwischen Produzenten und Transporteuren erfolgen kann.

44 Allerdings werden analytisch statt Ketten zunehmend Wertschöpfungs-Netzwerke betrachtet, da Unternehmen tatsächlich in ein Geflecht aus WK eingebunden sind, was sich auch in einer zunehmend kollektiven Risikowahrnehmung spiegeln dürfte, vgl. Engelhardt-Nowitzki et al. 2010. Auch der Ansatz der econophysics kombiniert traditionelle Input-Output-Analysen mit netzwerktheoretischen Methoden, vgl. z. B. Amador 2018., Vf. Bereits Svensson 2000 fordert einen holistischen, die ganze WK überspannenden Ansatz zur Erfassung von Vulnerabilitätsquellen, statt nur die direkten Lieferanten einzubeziehen (741). Bugert/Lasch 2018 zeigen, dass $90 \%$ der durch sie untersuchten Supply Chain Risiko-Modelle zumindest mehr als nur den direkten Partner im Netzwerk als Risikoquelle berücksichtigen (25). 45 Gereffi et al. 2005 nennen neben materialbezogenen Unternehmen in der Halbleiterindustrie auch IT-Unternehmen als Beispiele für Unternehmen, die die Wettbewerbsparameter im Markt bestimmen. 46 Wagner et. al. 2010. 


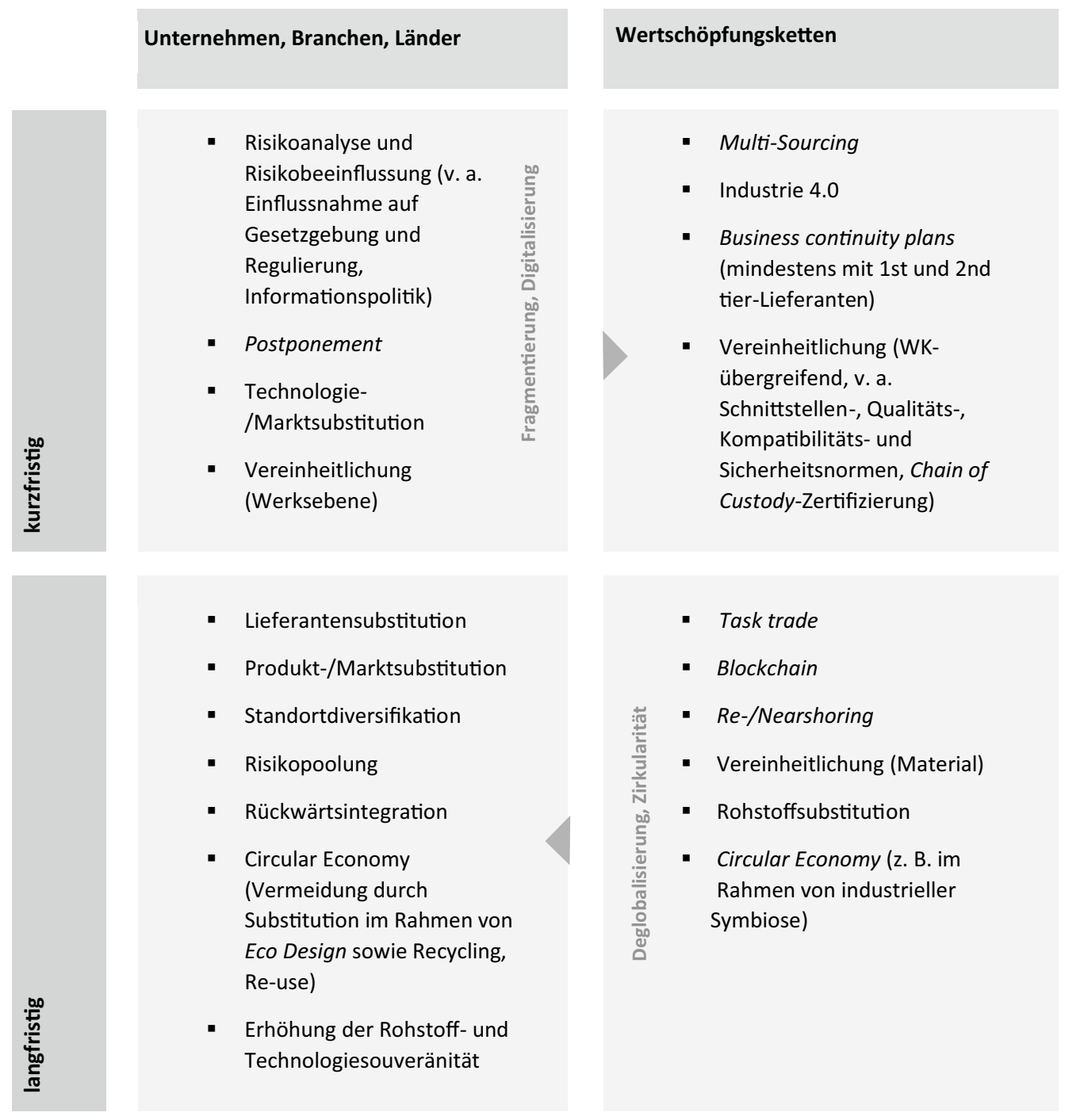

Abb. 7: Maßnahmen-Matrix der Governance von Vulnerabilität Quelle: eigene Darstellung

ken. ${ }^{47}$ Vulnerabilität kann ex ante durch Vermeiden riskanter Standorte reduziert werden und indem Einfluss darauf genommen wird, welche Wahrscheinlichkeitsverteilung den Risiken unterliegt (risk shaping), beispielsweise durch Mitgestaltung der nationalen und internationalen Gesetzgebung und Regulierung. ${ }^{48}$ Auch das Verschieben (postponement) von Produktionsstufen und Investitionen auf einen späteren Abschnitt der Wertschöpfungskette und damit einen Zeitpunkt, zu dem die Unsicherheit (beispielsweise über Nachfrageparameter) geringer ist, reduziert Vulnerabilität. ${ }^{49}$ Ex post kann eine Streuung von Risiken (risk pooling) in der Lieferantenkooperation erfolgen und in einigen Fällen kann dies sogar zu einer Verbesserung der Ausgangslage führen, wie die Übernahme des Marktanteils eines insolvent gewordenen Wettbewerbers. ${ }^{50}$ Eine stärker lokale Ausrichtung der Lieferantenbasis (nicht zu verwechseln mit Single-Sourcing) reduziert langfristig Vulnerabilität, nimmt aber auch erreichte Produktionskostensenkung wieder zurück..$^{51}$ Abbildung 8 zeigt die Interdependenz von Branchen, Technologien, Werk- und Rohstoffen in der Vulnerabilitätsanalyse, beispielhaft sei das Kalkül aus der Sicht eines Unternehmens gezeigt. 


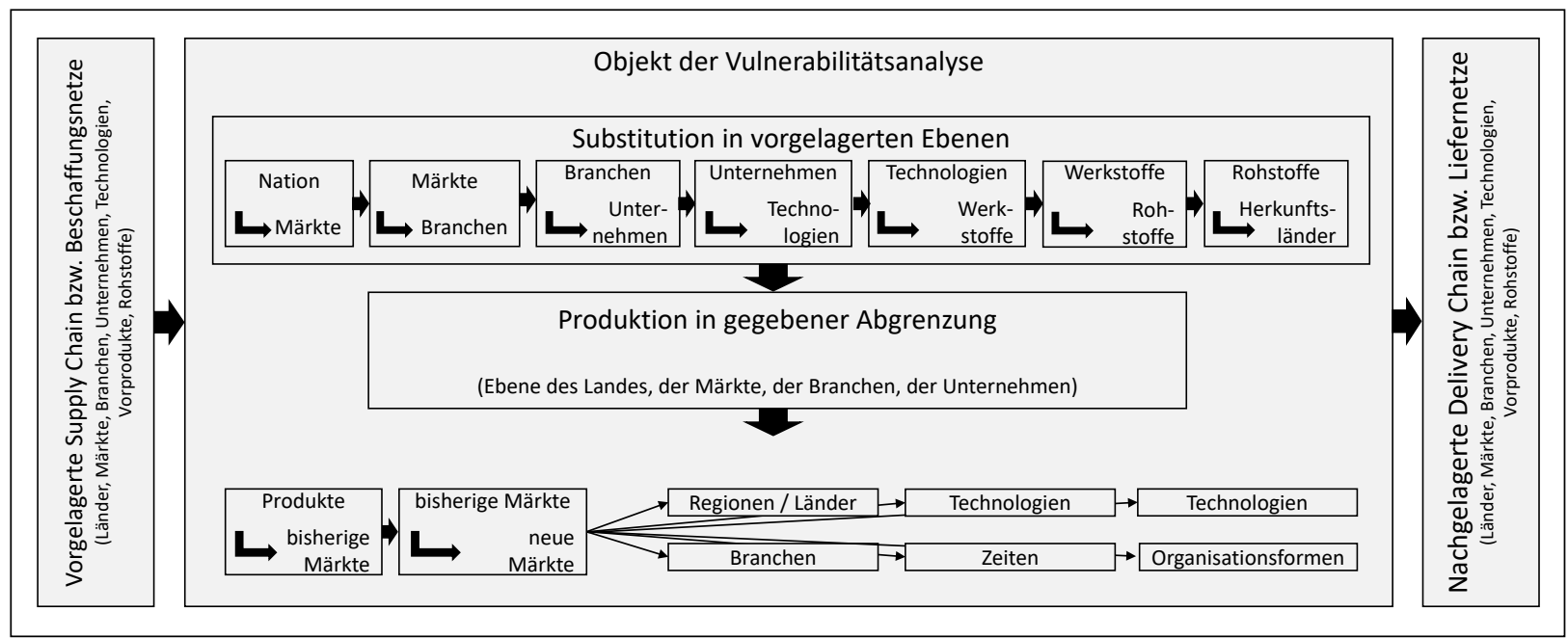

Abb. 8: Vulnerabilitätsanalyse von Unternehmen in Wertschöpfungsketten Quelle: eigene Darstellung

Seine grundlegende Risikoposition bezieht das Unternehmen aus der Position in einem Land und einer Branche, die wichtige erste Bestandteile des Ratings sind. Im Fall von Engpässen auf der Beschaffungsseite hat das Unternehmen die Möglichkeit, erst andere Technologien, dann andere Werkstoffe, schließlich andere Rohstoffe einzusetzen. Auf der Absatzseite würde ein plötzlicher Schock zur Überprüfung des Absatzkanals und schließlich verschiedenen Innovationsoptionen führen, die teilweise in der Innovationstheorie Schumpeters abgedeckt sind, der Produkt, Markt, Prozess, Organisation und Lieferquelle als systematische Felder von (häufig disruptiv erzwungener) Innovation sieht. ${ }^{52}$

Über qualitative oder quantitative Input-Output-Techniken kann das Wandern von Schocks verfolgt werden, um anschließend, beispielsweise über Monte-Carlo-Simulationen, auf Sektor- oder Unternehmensebene die Vulnerabilität auszuweisen. Übliche Indikatoren erfassen den Unternehmenswert, der bei einer bestimmten, akzeptierten Insolvenzwahrscheinlichkeit - beispielsweise 2 Prozent - im Risiko steht, oder den Wert des Kapitals, das zuzuführen wäre, um ein bestimmtes Risiko auszugleichen. ${ }^{53}$

Die Fähigkeit zur Gestaltung der Konfiguration von Wertschöpfungsketten wird durch Marktmacht bestimmt, so können Branchenführer oder fokale Unternehmen in Netzwerken auf das Risiko- und Ertragsprofil stärke-

52 Schumpeter 1912, 1927, 1942.

53 Die Finanzwirtschaft erfasst dies als Value-at Risk oder als RiskAdjusted Capital. ren Einfluss nehmen als periphere Folgeunternehmen, daneben bestimmen Eigenschaften der Transaktion (Komplexität, Kodifizierbarkeit durch Normen/Standards), die Möglichkeit zur Lieferantensubstitution (langfristig). Im Folgenden wird Governance von Vulnerabilität beispielhaft für zentrale Handlungsfelder auf der Ebene von Wertschöpfungsketten aufgezeigt (rechte Hälfte der Abbildung 7).

\subsection{Wertschöpfungsketten-Ebene: Industrie 4.0, task trade und Blockchain}

Wertschöpfungskettenübergreifend wird zur Vulnerabilitätssenkung oft ein mit Lieferanten gemeinsam entwickelter Notfallplan (business continuity plan) empfohlen. ${ }^{54}$ Nicht für alle Unternehmensgrößen ist eine redundante Vergabe an mehrere Lieferanten (Multi-Sourcing) angezeigt. Der Risikosenkung durch Diversifikation aufgrund einer kleinteiligen Vergabe von Aufgaben(teilen) an verschiedene Lieferanten stehen gestiegene Kontroll- und Koordinationskosten gegenüber. Angesichts hoher Koordinationskosten wird Unternehmen im Preiswettbewerb (unter der Voraussetzung einer engen Lieferantenbindung) zumeist ein Single-Sourcing empfohlen. Dagegen

54 Jüttner/Maklan 2011 unterscheiden beim Supply Chain Risk Management zwischen Maßnahmen, die Vulnerabilität senken (z.B. Meiden bestimmter Regionen) und damit die Auftretenswahrscheinlichkeit disruptiver Ereignisse reduzieren und solchen, die Resilienz erhöhen, d.h. die Ereignisfolgen abschwächen, 249. 
sollten im Qualitäts- bzw. Zeitwettbewerb stehende Unternehmen eine Wertschöpfungskette mit redundanten Lieferanten (Multi-Sourcing) etablieren, denn geringere Vulnerabilität, die vom Markt wegen höherer Verlässlichkeit auch honoriert wird, gleicht dann die gestiegenen Koordinationskosten aus. ${ }^{55}$

Durch die umfassende Unterlegung von Material, Komponenten und Produkten mit Produktions- und Nutzungsdaten in der Industrie 4.0 werden zudem die Informationsflüsse kontrollierbarer, vorausgesetzt, es besteht eine Vereinheitlichung von Datenformaten und Schnittstellen (siehe näher Abschnitt 3.3). So werden Chain of custody-Zertifizierungsdaten beim Material bzw. Produkt selbst hinterlegt, um einen lückenlosen Herkunftsnachweis von Material zu führen, wie er beispielsweise schon für Konfliktmetalle etabliert ist. Dies ermöglicht es auch Automobilunternehmen, sicherzustellen, dass Originalteile verbaut werden sowie datenbasierte Geschäftsmodelle basierend auf dem Nutzerverhalten über den Lebenszyklus des Fahrzeugs zu etablieren. Dass durch Industrie 4.0 auch die Anfälligkeit kritischer Infrastrukturen steigt, wird unter dem Begriff Technologiesouveränität wieder aufgegriffen.

Wertschöpfungsketten-Governance richtet sich nicht mehr auf Zwischenprodukte oder Produkte, sondern auf Abschnitte der Wertschöpfungsketten (tasks). ${ }^{56}$ Unter den Vorzeichen des task trade wird beispielsweise die Aufgabe des Rotordesigns für eine Flugzeugturbine durch den Hersteller selbst übernommen, einzelne Aufgaben in der Produktion der Turbine dagegen ausgelagert. Die Governance von Vulnerabilität würde sich dann auch auf Aufgaben statt auf einzelne Kontraktpartner richten müssen. Wird die Turbine, um im Beispiel zu bleiben, jedoch durch 3 D-Druck hergestellt (wie im Fall der smarten Turbinen von General Electric), weist sie eine geringe Kontraktintensität auf, was die Kontrollkosten senkt. Lieferten für die Produktion der Einspritzdüse für General Electric-Triebwerke früher 20 Lieferanten Komponenten, ist heute nur noch ein Lieferant für das Metalloxidpulver notwendig, das im 3 D-Druck eingesetzt wird. ${ }^{57}$ Marktmacht wird sich durch Industrie $4.0 \mathrm{zu}$ Lieferanten des Metalloxidpulvers

55 Neureuther/Kenyon 2009, 259 als Ergebnis einer Reliabilitätsanalyse verschiedener Wertschöpfungskettentypen.

56 Grossman/Rossi-Hansberg 2012.

57 Vgl. Märki, Martina/Kasielke, Nicole: Innovation ist ein Balanceakt, Interview mit den Professoren Mirko Meboldt und Torbjörn Netland, ETH Globe 01.12.2017; https://ethz.ch/de/news-und-veranstal tungen/eth-news/news/2017/12/innovation-ist-ein-balanceakt.html. Die aktuelle Häufung von Ausfällen bei GE-Triebwerken in Boeing Dreamlinern scheint auf Designfehler und nicht auf die Produktionstechnik zurückzugehen. hin verschieben, da dieses qualitätskritisch ist, begleitet von strukturellen Veränderungen im wertschöpfungsintensiven produzierenden Gewerbe. ${ }^{58}$ Durch die weltweite Restrukturierung der Industrie zu Industrie 4.0 könnten daher Re-shoring und Near-shoring zunehmen..$^{59}$ Vulnerabilität sinkt zugleich dadurch, dass die Kontrollintensität des nunmehr homogenen Zwischenprodukts geringer ist als bei jeder der einzelnen Komponenten zuvor. Kritischen Infrastrukturen inhärente Risiken, die neben Verteidigung und Sicherheit auch Kernkompetenzen der Volkswirtschaft berühren, kann die Blockchain-Technologie reduzieren. ${ }^{60}$ Eine Blockchain (wörtlich Block-Kette) ist eine Datenbank, in der sich eine valide Transaktion (Block) an die andere reiht wie die Glieder einer Kette und jeweils alle vorangegangenen Transaktionen bestätigt. ${ }^{61}$ Durch Blockchains wird jede Transaktion, beispielsweise ein Vertrag oder eine Rechnung in einer Lieferkette, durch einen manipulationssicheren Datensatz erfasst (vergleichbar dem mittelalterlichen Kerbholz, von dem jeder Vertragspartner eine Hälfte erhält, nur ohne die physische Komponente). Für Unternehmen kann Blockchain-Technologie Monitoring-Kosten und damit Vulnerabilität senken. Das geht über bisherige Maßnahmen zur Überwachung von Wertschöpfungsketten hinaus, wie den Einsatz molekularer Materialmarker als Herkunftsnachweis und die RFIDTechnologie zur Rückverfolgung von Zwischen- und Endprodukten in der Lieferkette. Sowohl Verkäufer als auch Käufer können alle Transaktionsdaten überprüfen und Änderungen (Verlust, Beschädigung) von Gütern durch physisches Echtzeit-Tracking manipulationssicher ermitteln. ${ }^{62}$ Kosten sinken, da der Bedarf an vermittelnden Dritten (z. B. Notare, Gerichte) sowie an Dokumentation reduziert wird. Der Einsatz der Blockchain-Technologie in Wertschöpfungsketten steht dessen ungeachtet noch am Anfang. Eine Befragung der HTWK Leipzig unter 132 deutschen Unternehmen überwiegend aus der Industrie hat ergeben, dass zwei Drittel der Unternehmen noch keine Blockchain nutzen, bei weniger als 4 Prozent gibt es

58 Simulationen zeigen, dass im produzierenden Gewerbe Arbeitsplätze verloren gehen werden, dies aber mit einem parallelen Aufwuchs v. a. bei Dienstleistungen einhergeht, Wolter et al. 2016.

59 Runde/Bandura/Hammond 2019, 35.

60 Das Konzept der Kernkompetenzen von Prahalad/Hamel 1990 wird zwar meist auf Unternehmen oder Netzwerke und Cluster angewendet, es kann aber argumentiert werden, dass etwa eingebettete Systeme wie der Airbag und die Vernetzung eingebetteter Systeme wie der Bremsassistent für Autos schwer imitierbare, nachhaltige Wettbewerbsvorteile für die Volkswirtschaft generieren, Scheer 2013, 10.

61 Deubel/Moormann/Holotiuk 2017, 830.

62 Min 2019, $42 \mathrm{f}$. 
eine solche mindestens auf Prototypenniveau, nur knapp 7 Prozent erkunden konkrete Anwendungsszenarien, jedoch erwarten 80 Prozent eine zukünftige Nutzung. ${ }^{63}$

\subsection{Vereinheitlichung als Strategie der Wertschöpfungsketten-Neuordnung}

Ein weiteres Instrument zur Senkung von Vulnerabilität sind die verschiedenen Formen der Vereinheitlichung, also Typung auf der Betriebsebene, Industriestandards auf der Ebene der Kooperation zwischen Unternehmen und Normen im nationalen bzw. supranationalen Kontext. Vor allem letztere spielen aufgrund öffentlicher Gewährleistung eine wichtige Rolle. ${ }^{64}$ Ein direkter Zusammenhang zwischen Normen und Vulnerabilitäts-Governance besteht bei Qualitätsnormen, die ihren Ursprung im US-amerikanischen und britischen Verteidigungssektor der 60er und 70er Jahre des 20. Jahrhunderts haben und beinahe direkt in Qualitätsmanagement-Normen heutiger privatwirtschaftlicher Unternehmen übernommen wurden. ${ }^{65}$ Im Bausektor und der chemischen Industrie steht Sicherheit (bezogen nicht auf security, sondern auf safety) im engen Zusammenhang mit Nachhaltigkeit. Zu nennen sind die ISO-Normen zu Carbon Footprint und Water Footprint, die den Aufbau nachhaltiger Wertschöpfungsketten fördern, indem sie Vulnerabilität gegenüber Umweltrisiken senken. ${ }^{66}$ Seit 2016 wird auch die Rückverfolgbarkeit von Lieferketten durch eine Norm unterstützt (ISO 22095).

Die verschiedenen Vereinheitlichungsformen, insbesondere die Normung, verweisen auf Informationsflüsse entlang der Wertschöpfungskette und wie diese durch IPR-Strategien gesteuert werden können. Insbesondere Patente spielen dabei eine wichtige Rolle, ist ihre Nutzung doch im Rahmen der Normung zu einem angemessenen Preis zu ermöglichen. Hierzu wird sich der Patentinhaber nur dann entschließen, wenn er glaubt, dass seine Technologie dadurch eine verstärkte Verbreitung findet.

63 Meinert/Müller 2018.

64 Das Erfüllen einer Norm stärkt die Position des Herstellers im Falle entsprechender Schadens- oder Qualitätsklagen. Zudem stellt die Normung eine Plattform dar, die eine starke Garantiefunktion für die Verlässlichkeit bei der Absatzplanung ausübt; vgl. Blum/Bahke/ Eickhoff 2002.

65 Gibbon/Henriksen 2011, 131.

66 Navaz/Linke/Koç 2019, 79. Die genannten Normen dienen der Abbildung von Wasserverbrauch und -qualität in einer Ökobilanz (ISO 14046, Water Footprint) und dem Nachweis der Treibhausgas-Emissionen über den Lebenszyklus eines Produkts hinweg (ISO 14067, Carbon Footprint).
In der Literatur zu internationalen Wertschöpfungsketten werden Informations-Transaktionskosten (die durch Normung gesenkt werden können) als zunehmend wichtiges Forschungsfeld betrachtet, da ihr Rückgang globale Wertschöpfungsketten erst hervorgebracht hat. ${ }^{67}$

Über diese unmittelbaren Zusammenhänge hinaus sollen Normen im Folgenden als Informationsträger und in dieser Eigenschaft nicht nur als Ergebnis, sondern als Mittel zur Restrukturierung von Wertschöpfungsketten betrachtet werden. ${ }^{68}$ In globalen Wertschöpfungsketten sind Normen Substitute für das kostenverursachende Monitoring der Qualität von Zwischenprodukten, d.h. harmonisierte Normen senken Handelskosten. Dagegen haben nicht harmonisierte Normen einen handelsbeschränkenden Effekt, was sich v. a. für kleine Unternehmen in Ländern mit geringem Technologisierungsgrad nachteilig auswirkt. ${ }^{69}$ Dem steht gegenüber, dass kleine und mittelständische Unternehmen aufgrund ihrer geringen Einbindung in globale Wertschöpfungsketten (beispielsweise überwiegend lokale Beschaffung, Kleinserienproduktion) insgesamt einer geringeren Vulnerabilität ausgesetzt sind, als Großunternehmen oder Unternehmen in stark gekoppelten und interaktiven Prozessindustrien wie Chemie, Pharmazie, Stahlproduktion und Plastik. ${ }^{70}$ Normen wirken sich unterschiedlich über die Wertschöpfungskette hinweg aus. So verstärken Qualitätsnormen (beispielsweise ISO 9000 ff.) die Koordination über den Markt, während eine zunehmende Dichte an Umwelt-/ Lebensmittel- und Arbeitsstandards im Lebensmittel-Einzel- und Großhandel keine Zunahme marktlicher Koordination, sondern ein rigoroseres Lieferantenscreening zur Folge hat. ${ }^{71}$ In diesem Fall steht einem starken Großhandelsunternehmen eine vulnerable Lieferantenbasis (Lebensmittellieferanten, u. a. Agrofood-Betriebe) gegenüber. Die Marktmacht ermöglicht es dem Handelsunternehmen beispielsweise, glaubwürdig mit Auslistung zu drohen, wenn es zudem Eigenmarken als Substitute einsetzen kann. Es bilden sich kaptive Wertschöpfungsketten, also solche, aus denen Teilnehmer nicht ohne erhebliche Schäden ausbrechen können. ${ }^{72}$ Normen und Zertifizierung (beispielsweise FSC in der nachhaltigen

67 Escaith 2017, 108, Blum 2018, 737.

68 Ponte/Gibbon/Vestergaard 2011, 8. Auch Bugert/Lasch 2018, 4 erwähnen supply chain design zur Senkung von Vulnerabilität.

69 Escaith 2017, 103. Nadvi 2008 nimmt ebenfalls ein matching zwischen Normen und Marktstruktur vor.

70 Wagner/Neshat 2012, 2881 u. 2887.

71 Nadvi 2008, $332 \mathrm{f}$.

72 Gereffi 2011. 
Forstwirtschaft) ${ }^{73}$ verstärken also in Konsumenten-Wertschöpfungsketten die Marktmacht von lead firms (Handel). ${ }^{74}$ Compliance-Kosten werden auf Lieferanten (Holzproduzenten) überwälzt, für die die Zertifizierung zum Selektionskriterium im Markt wird, wobei nur das Erreichen kritischer Masse (Kooperation) den Marktaustritt verhindern kann. ${ }^{75}$ Wie sich insgesamt Vereinheitlichung auf die Vulnerabilität auswirkt kann nur am konkreten Beispiel überprüft werden. Im vierten Abschnitt wird die Wettbewerbsrelevanz der Vereinheitlichung von Material und Komponenten am Beispiel der Wertschöpfungsketten von Hochleistungsmagneten aufgezeigt.

\subsection{Schließen von Wertschöpfungsketten in der Circular Economy}

Auch Recycling und Wiederverwendung (als Vorboten einer Circular Economy) schließen Wertschöpfungsketten bzw. verbessern die Position an den Rändern der „Lachkurve“ (siehe Abbildung 3 und unterer Teil von Abbildung 7). Dabei kann Unsicherheit in Quantität und Qualität der Inputfaktoren durch einen Zustrom an Rezyklaten (Sekundärinputs, also nach der Nutzungsphase wiedergewonnene Ressourcen) reduziert werden. ${ }^{76}$ Damit ist gemeint, dass Unternehmen unabhängiger von Inputqualität und Preisen ihrer Lieferanten werden, wenn sie z.B. Neuplastik durch qualitativ ausreichende Rezyklate ersetzen oder Seltene Erden statt im Direktbezug aus China im Wege des Recycling von Elektronikgeräten oder

73 Vgl. Webseite des FSC Deutschland (2019): Die Produktkettenzertifizierung. Vom Wald ins Wohnzimmer; https://www.fsc-deutschland. de/de-de/zertifizierung/produktkettenzertifizierung-coc.

74 In Übertragung des Konzepts des Verkäufer- bzw. Käufermarktes auf produzenten- bzw. konsumentengetriebene Wertschöpfungsketten unterscheiden Gereffi 2011 und Gereffi/Lee 2012 ProduzentenWertschöpfungsketten mit hohem Technologiegehalt und Spezialisierung auf Nischen und Konsumenten-Wertschöpfungsketten mit hohen Margen, standardisierten Produkten sowie effektivem Vertrieb. 75 Am Beispiel der Forstwirtschaft Mexikos zeigt Klooster 2011 auf, dass die FSC-Zertifizierung per se keinen Zugang zu globalen Wertschöpfungsketten erzeugte (u. a. aufgrund zu geringer Liefermengen und fehlender Preiswettbewerbsfähigkeit), jedoch gelang durch Einkaufs- und Vertriebsgemeinschaften das Upgrading von der Holz- zur Möbelproduktion.

76 Blum 2018, zum Recycling Stindt/Sahamie 2014, 281. Es wird zwischen Primär-Recycling (Abfälle aus der Produktion von Halbfertigerzeugnissen, z. B. Seltene Erdmetalle aus der Produktion, die wieder eingeschmolzen werden) und Sekundär-Recycling (Rückgewonnene Seltene Erden aus Fertigerzeugnissen wie Windrädern und Elektroautomobilen am Ende ihrer Nutzungsdauer) unterschieden, vgl. Keilhacker/Minner 2017, 354. gebrauchten Magneten gewinnen können. Recycling ist etabliert für Produktionsabfälle, jedoch in einigen Branchen für die wesentlich wichtigeren Konsumentenabfälle erst im Aufbau. Dabei ist zu berücksichtigen, dass erhebliche Branchenunterschiede bestehen. So ist in der Kunststoffindustrie die Wiederverwendung von gebrauchtem Polymer-Material durch Beimischung von Originalmaterial nicht möglich, unproblematisch ist dies dagegen in anderen Prozessindustrien. ${ }^{77}$ Dies kann im Rahmen der Circular Economy mit industrieller Symbiose verbunden werden. Diese kleinen Industriekreisläufe ersetzen zum Teil die zuvor global etablierten Wertschöpfungsketten, jedoch mit dem zusätzlichen Anspruch, materiell und energetisch geschlossene Stoffkreisläufe herzustellen. Ein vollständiger Ersatz ist gleichwohl aufgrund der geographisch unterschiedlichen Ressourcenverfügbarkeit (Lagerstätten) sowie der physischen Grenzen der technologischen Substitution nicht möglich. Langfristig sind eine Recyclinginfrastruktur sowie eine Kopplung zwischen verarbeitender Industrie, Chemieunternehmen und Verwertern einzurichten. Dies bietet das Potenzial, Energie sowie Materialeinsatz zu reduzieren und damit industrieübergreifend Vulnerabilität zu senken, indem Industriezyklen verlängert werden. Dies kann mit Industrie 4.0 und Cybersicherheit für eine höhere Rohstoff- und Technologiesouveränität verbunden werden, wie im Folgenden und in Abschnitt 4 noch näher erläutert wird.

Beispiele für Rohstoffsouveränität durch Substitution sind der Ersatz petrobasierter durch biobasierte Kunststoffe (wodurch zugleich die industrielle Abhängigkeit von Erdölimporten reduziert wird) und die Substitution Seltener Erden. Beide sind Gegenstand laufender Forschung, weil die Eigenschaften der Substitute häufig nicht an die des etablierten Kunststoffs/des Selten-Erdbasierten Magneten heranreichen. Das Fraunhofer-Leitprojekt Kritikalität Seltener Erden hat Ansatzpunkte für eine Reduzierung der Ressourcenabhängigkeit durch Design (inkl. Normung), Recycling und Substitution aufgezeigt und so einen Beitrag zur Vulnerabilitätssenkung geleistet. ${ }^{78}$ Es wird erwartet, dass aufgrund der relativ hohen Magnetmasse von 1-3 kg in Traktionsmotoren von Elektroautos sowie des hohen Wertes des enthaltenen Technologiemetalls Dysprosium sich (im Gegensatz zu Kleinelektrogeräten) ein Sammel- und Recyclingsystem für das Sekundärrecycling dieser großen Magnete entwickeln wird, die

77 Stindt/Sahamie 2014, 284.

78 Wehrspohn 2017. Vgl. grundlegend zu Normen und materialintensiven Wertschöpfungsketten Labucay 2018. 
mittelfristig in großer Menge aus gebrauchten Elektroautos zur Verfügung stehen werden. ${ }^{79}$

Wurde Technologiesouveränität zunächst verstanden als Verfügbarkeit und Beherrschbarkeit von Sicherheitstechnologien, wird sie aktuell mit der Sicherung kritischer Infrastrukturen verbunden. Bei Komponenten der 5G-Infrastruktur etwa geht im Vergleich zu Cyberspionage die höhere Vulnerabilität wohl von der Möglichkeit eines Kill-Switch aus, dem Lahmlegen von Kommunikationsinfrastrukturen. In der Industrie 4.0 geht Vulnerabilität von smarten Produkten, Produktions- und Lieferketten aus. ${ }^{80}$ Dieses ist im Prüfverfahren nach dem Außenwirtschaftsrecht zu berücksichtigen, um die Technologiesouveränität über den Kernbereich des 5G-Netzes zu erhalten.

Wie eine Governance von Vulnerabilität konkret aussehen kann, wird im Folgenden am Beispiel der besonders vulnerablen Wertschöpfungskette von Hochleistungsmagneten aufgezeigt, hier wird auch die erwähnte Schließung der Wertschöpfungskette noch einmal aufgegriffen.

\section{Wertschöpfungsketten von Hochleistungsmagneten}

Die am häufigsten eingesetzten Hochleistungsmagnete sind Neodym-Eisen-Bor $\left(\mathrm{Nd}_{2} \mathrm{Fe}_{14} \mathrm{~B}\right)$-Magnete. Diese wurden 1982 zeitgleich durch General Motors und Sumitomo Special Metals entwickelt und gelten heute als stärkste verfügbare Permanentmagnete. ${ }^{81} \mathrm{Am}$ Beginn der Wertschöpfungskette von Hochleistungsmagneten stehen Seltene Erden. Diese sind, wie früher ausgeführt, „raumrelevant“, da ihre Gewinnungsindustrien an wenige Standorte weltweit gebunden sind, auch weil die prekären Umstände ihrer Gewinnung nur in einem schwachen Regulierungsumfeld Bestand haben konnten. ${ }^{82}$

Die Wertschöpfungskette von Hochleistungsmagneten ist als Beispiel für Vulnerabilitäts-Governance geeignet, da sie an ihrem upstream-Ende der Rohstoffgewinnung und -aufbereitung schon seit einigen Jahrzehnten etabliert und die Marktstruktur konzentriert ist. Wenngleich Amerika und Australien Erschließung und Betrieb vormals

79 Glöser-Chahoud/Tercero Espinoza 2015, $69 \mathrm{f}$.

80 Vgl. Bundesverband der Deutschen Industrie e. V. - BDI: Sicherheit durch technologische Souveränität!, Webseite des BDI, 29.10.2015; https://bdi.eu/artikel/news/sicherheit-durch-technologischesouveraenitaet/. Vgl. auch Stefan Häberli: Sprechen wir über Huawei, Neue Zürcher Zeitung, 14.03.2020.

81 Voncken 2016, 97.

82 Blum/Schmid 2015. geschlossener Selten-Erd-Minen wieder aufgenommen (teilweise auch wieder eingestellt) haben, konnten sie bis Mitte der 2010er Jahre nur geringe Teile der schweren Seltenen Erden fördern, so dass das Monopol Chinas bei den schweren Seltenen Erden weiter Bestand hatte. Abbildung 9 verdeutlich aber, dass sich dies inzwischen geändert hat, und der Marktanteil Chinas relativ gesehen auf rund $60 \%$ gefallen ist - absolut betrachtet aber etwa konstant bleibt. Es sind vor allem Australien und Burma, die mit einer Förderung von aktuell knapp 50.000 Tonnen die Expansion der Nachfrage stillen konnten. Auch sind die USA mit aktuell rund 40.000 Tonnen pro Jahr seit Mitte des letztens Jahrzehnts wieder ein relevanter Förderstandort.

Weiterhin weisen die downstream-Industrien der Hochleistungsmagnete noch eine hohe Dynamik auf. Bei NdFeB-Magneten könnte sich wiederholen, was mit ihren auf Aluminium, Nickel und Kobalt (AlNiCo) bzw. Kobalt und Samarium (SmCo) basierenden Vorgängern geschah. Diese waren noch bis in die sechziger Jahre des letzten Jahrhunderts die gängigsten Hochleistungsmagnete, bis die Kongokrise die Entwicklung der neuen Hochleistungsmagnete auf Basis Seltener Erden beschleunigte.

Eine große Rolle zur Erlangung einer erhöhten Technologie- und Rohstoffsouveränität kommt der Vereinheitlichung durch Normen und Industriestandards zu. Die internationale Normungsorganisation ISO stellt mit Blick auf die Selten-Erd-Wertschöpfungskette fest: "There is a poor understanding from a life-cycle perspective of the rare earth value chain, from mine to product, and the overall efficiency of the supply chain." ${ }^{\text {83 }}$ Normen sind hier als Qualitätssignal Voraussetzung für das Entstehen funktionierender Märkte und zur Senkung von Vulnerabilität in der Wertschöpfungskette. Die Norm WK 4403 etwa wirkt als Signal der Zahlungsbereitschaft des Marktes für einen bestimmten Reinheitsgrad der Selten-Erd-Oxide, da Produzenten Seltener Erden Sicherheit über die Zusammensetzung und Qualität erhalten und die aus Produktionsschwankungen resultierende Vulnerabilität ihrer Wertschöpfungskette senken können. ${ }^{84}$

83 ISO (2016). Pell et al. 2019, 65 f. unterscheiden die Stufen Abbau, Aufbereitung, Abfallmanagement und Transport Seltener Erden (upstream) sowie Auftrennung und Verarbeitung der Oxid-Verbindungen zur Gewinnung der einzelnen Seltenen Erden (cradle-to-gate) und deren Weiterverarbeitung zu Endprodukten (downstream).

84 WK 4403 der American Society for Testing and Materials, die den vollständigen Titel trägt: Test Method for Identification and Quantification of Rare Earth Elements (REEs) in Rare Earth Materials (REMs) by X-Ray Fluorescence Spectrometry, vgl. Rare Earth Elements, in: ASTM Standardization news, January/February 2014; https://www. astm.org/standardization-news/?q=update/rare-earth-elements-jf14. html. 


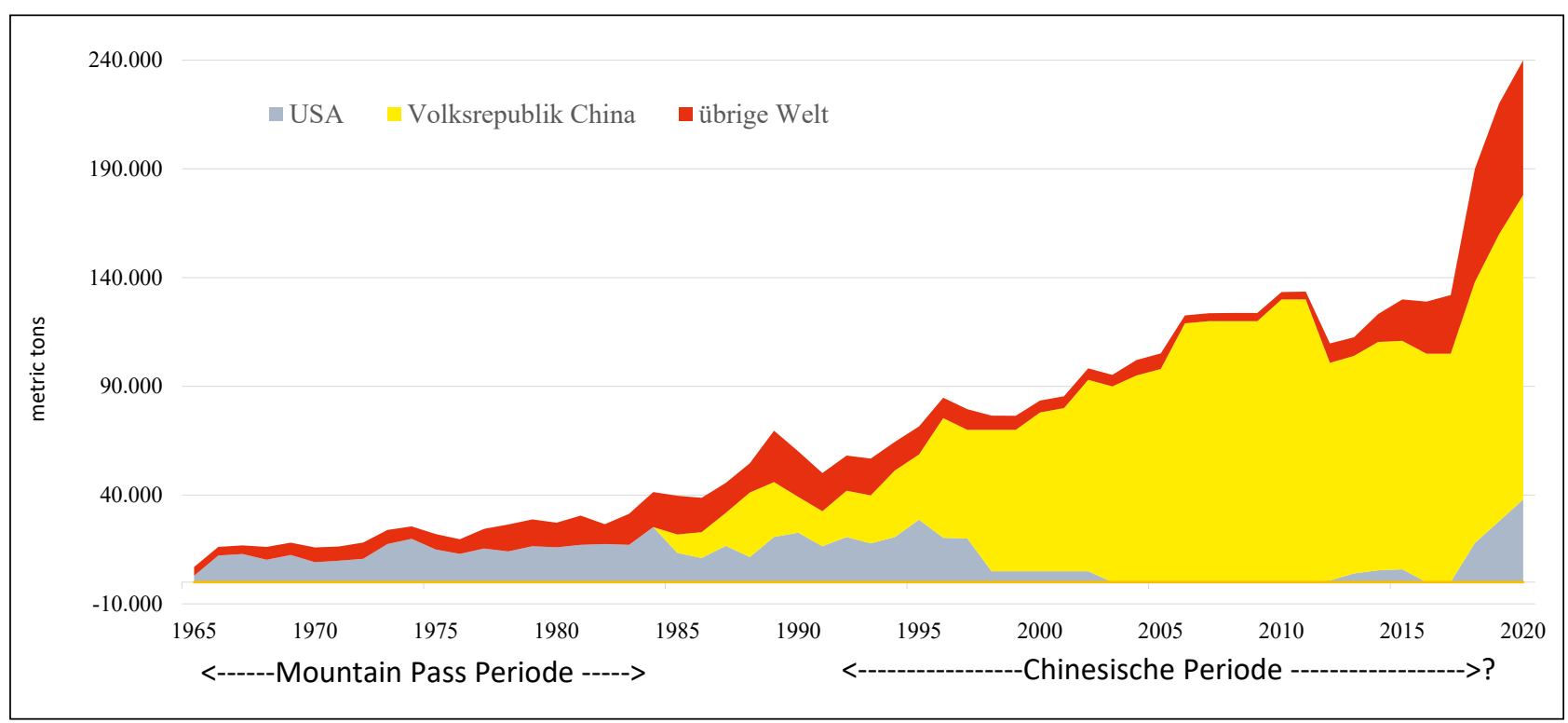

Abb. 9: Die bröckelnde Dominanz Chinas bei der Förderung Seltener Erden Quelle: eigene Darstellung nach Daten des United States Geological Survey, https://www.usgs.gov/centers/nmic/rare-earths-statisticsand-information

Modularer Aufbau von Magneten, der durch Normung abgesichert wird, schlägt zudem die Brücke zur Circular Economy. Das Investitionsrisiko von auf Permanentmagneten basierenden Technologien (beispielsweise Elektromotoren in Automobilen und Windgeneratoren) sinkt, wenn zukünftige Nutzungspfade erschlossen werden und eine Wiederverwendungskaskade etabliert wird, die von höherwertigen Magneten, beispielsweise in der Unterhaltungselektronik, bis zu einfachen Industriemotoren reicht, was aber wiederum Normung und Standardisierung der Magnete voraussetzt. ${ }^{85}$ Hier haben Normen vor allem die Funktion, die Vielfalt an Bauformen und Zusammensetzung der Magnete zu beschränken, um ihre Dekonstruktion wirtschaftlich werden zu lassen. Die Wiederverwendung von Elektromotoren aus Automobilen findet jedoch noch kaum statt, da Normen für einen einheitlichen Rotoraufbau fehlen. ${ }^{86}$ Die Vulnerabilitätssenkung ist dann vorrangig durch Substitution zu erreichen.

Im Lichte des Schocks der Exportbeschränkung Seltener Erden im Jahre 2010 (vgl. Abbildung 5) wurde erstmals umfassend die Effektivität von proaktiven Strategien (Substitution, Neueröffnung von Minen, Forschung und Entwicklung) und reaktiven Strategien (Recycling) zur Senkung von Vulnerabilität in der Wertschöpfungskette von Hochleistungsmagneten in zehn Ländern und 18

85 Blum/Schmid 2015.

86 Walachowicz et al. 2014.
Branchen bewertet. Produzenten von Fertigerzeugnissen (beispielsweise von Automobilen, Windrädern) können danach am effektivsten Substitution zur Senkung von Vulnerabilität einsetzen, gefolgt von Sekundär-Recycling (also dem Einsatz von aus Altmagneten und -sensoren zurückgewonnenen Seltenen Erden), jedoch erst ab einer 30 Prozent-Recyclingquote und vorhandener Infrastruktur. Demgegenüber hatte die Angebotsausweitung durch neue Minen keinen Effekt auf den Preis Seltener Erden. Die Übernachfrage ließ sich bei gleichzeitiger Substitution nur verzögert abbauen. ${ }^{87}$

Schließlich ist Vulnerabilität durch Monitoring von Information entlang der Wertschöpfungskette zu begegnen. Der Informationsraum lässt sich durch geistige Eigentumsrechte (IPR) erschließen bzw. verteidigen. Hier ist die Vulnerabilität umso höher je komplexer die Produkte sind. Bei komplexer Technologie steigt das Risiko, mit Innovationen Patentverletzungsklagen ausgesetzt zu sein, was das Ignorieren von Patenten zunehmend selbst zu einer Quelle von Vulnerabilität macht. ${ }^{88}$ Die strategische Erschließung von Raum durch Patente wird deutlich, wenn man nicht die Herkunftsländer, sondern die Zielländer der Patentan-

\section{Keilhacker/Minner 2017.}

88 Lemley 2008 zeigt auf, dass in Branchen mit einer hohen Patentdichte Innovationsfähigkeit wider Erwarten nicht zum Erliegen kommt, da eine „Strategie“ des Ignorierens von Patenten verfolgt wird, die den Verzicht des Eigentümers auf die Anstrengung von (langen und kostenintensiven) Patentverletzungsverfahren einberechnet. 
meldungen betrachtet. Erst dieser Blick legt die Marktstrategien offen. Im Bereich der auf Hochleistungsmagneten basierenden Windenergie lag beispielsweise Japan unter den Ländern mit den häufigsten Patentanmeldungen um die Jahrtausendwende noch an dritter Stelle. Im Jahr 2011 hingegen liegt Japan hinter Europa, China und den USA sowie weiteren Ländern nur noch an sechster Stelle, auch da sich mit Korea ein neuer strategischer Player eingefunden hat. ${ }^{89}$ Die Zielländer spiegeln das Verwertungsinteresse der Patentanmelder, d. h. die aus ihrer Sicht zentralen Märkte für magnetbasierte Produkte. Diese TechnologieMarkt-Kombinationen sind auch zentral in die Governance von Vulnerabilität einzubeziehen, beispielsweise bei der Lieferantendiversifikation, der Neuansiedlung von Standorten und der IPR-Strategie (nicht immer durch Eigenentwicklung, sondern auch, um geeignete Patente für die Lizensierung zu ermitteln). Die enge Kopplung im Informationsraum bedingt zudem einen umfassenden Informationsaustausch. Für die Automobilindustrie wurde gezeigt, dass bei hoher Vulnerabilität (gemessen an Schwankungen der Produktionsauslastung, der Endkundennachfrage und der Liefertreue), umfassender Informationsaustausch in der Wertschöpfungskette, also nicht nur zwischen dem Endkunden und seinem direkten Lieferanten, die Termintreue in der gesamten Kette erhöht. Dadurch lässt sich Vulnerabilität entsprechend senken. ${ }^{90}$ Strategische Wertschöpfungsketten-Governance beinhaltet damit auch das Lernen durch den Informationsaustausch in der Wertschöpfungskette, was insbesondere für zentrale Unternehmen mit deren Kontrolle gleichzusetzen ist.

\section{Zusammenfassende Schlussfolgerungen}

Dieser Aufsatz hat den für die Governance von Wertschöpfungsketten zentralen Tatbestand der Vulnerabilität aus Unternehmens-/Branchen- und Länder-Sicht sowie aus der übergreifenden Wertschöpfungsketten-Sicht verdeutlicht und am Beispiel von komplexen Hightech-Produkten und Hochleistungsmagneten demonstriert. Es wurde deutlich, dass Rohstoffe und Technologien ins Zentrum strategischer Industrie- und Handelspolitik gerückt sind.

Vulnerabilität wurde zwar unter den Begriffen Risiko und Resilienz schon früher auf Wertschöpfungsketten bezogen. In diesem Artikel wurden am Beispiel einer

89 UNEP/EPO 2015, 51.

90 Yüzgülec/Witthaut/Hellingrath 2010, 83 f. u. 93.
Hochrisiko-Wertschöpfungskette beginnend bei Seltenen Erden bis zu Hochleistungsmagneten konkrete Vorschläge zur Governance von Vulnerabilität gemacht, die Kritikalität und Rohstoffstrategien berücksichtigen und von der Unternehmensebene bis zur globalen Ebene reichen. Aktualität gewinnt diese durch die aktuelle Wertschöpfungsketten-Disruption durch die Corona-Pandemie, das Zurückdrehen der Globalisierung durch Renationalisierung von Wertschöpfungsketten und mit Blick auf die sich abzeichnende Circular Economy.

Für die Governance von Vulnerabilität materialintensiver Wertschöpfungsketten ist zusammenfassend $\mathrm{zu}$ empfehlen, dass Großunternehmen und Unternehmen vulnerabler Prozessindustrien wie der Chemie - und Lebensmittelindustrie sowie materialintensiver Wertschöpfungsketten eine interorganisationale Diversifikation betreiben. Dazu zählt nicht nur die bereits angesprochene Beschaffungsdiversifikation, sondern auch die Diversifikation über technologisch breit abgestützte Patente und Kooperation entlang der Wertschöpfungskette sowie interindustrielle Forschung und Entwicklung.

Für Hochrisiko-Wertschöpfungsketten wie die der Seltenen Erden und ihre Zwischen- und Endprodukte erweist sich Substitution durch Recycling von Fertigerzeugnissen (Windräder, Motoren, etc.) oder petrobasierter durch biobasierte Kunststoffe ${ }^{91}$ als effektive Maßnahme zur Senkung von Vulnerabilität. ${ }^{92}$ Auch ein verändertes Produktdesign (wobei Normen eine wesentliche Rolle spielen), das mit reduzierten Anteilen Seltener Erden annähernd gleiche Leistung (von beispielsweise Elektromotoren) erzielt, senkt die Anfälligkeit. Hier haben das Leitprojekt Kritikalität Seltener Erden sowie das Total Design Management neue Ansatzpunkte aufgezeigt. ${ }^{93}$ Durch Vereinheitlichung, insbesondere durch Normung, kann die Austauschbarkeit erhöht werden. Dies wird auch durch neue Geschäftsmodelle ergänzt, beispielsweise durch ein Leasing der Magnete, die aufgrund vorgegebener Geometrien und Leistungsdaten von einem außer Dienst gestellten Windgenerator in eine neue Turbine eingesetzt werden können. Technologiezyklen werden so verlängert und Wertschöpfungsgewinne entlang technologischer Wettbewerbsfähigkeit neu verteilt.

91 Dadurch wird zugleich die industrielle Abhängigkeit von Erdölimporten reduziert

92 Vgl. erneut Keilhacker/Minner 2017, 356 u. 359. Vgl. zu Zielkonflikten, die hieraus für die Circular Economy resultieren, am Beispiel der Zirkularitätsbewertung von Bioplastik Labucay 2021.

93 Zum Leitprojekt Kritikalität Seltener Erden Vgl. Wehrspohn 2017; zum Total Design Management siehe Blum 2021. Vgl. hierzu auch die Ausführungen in den einzelnen Kapiteln bei Blum/Wehrspohn 2021. 
Richten sich gegenwärtige Maßnahmen zur Erhöhung der Technologiesouveränität noch vorrangig auf die Sicherung kritischer Infrastrukturen im Prüfverfahren nach dem Außenwirtschaftsrecht, ist auch an die Sicherung der industriellen Basis durch Schutz der wertschöpfungsintensiven produzierenden Industrie vor einer Digitalisierung zu denken, die Kernkompetenzen bedrohen kann. Hier kommt Normungsplattformen, die Informationsflüsse vereinfachen und schützen, Mindestqualitäten von Prozessen und Produkten definieren und Geschäftsmodelle absichern, eine hohe Bedeutung zu. Um Rohstoffsouveränität zu erreichen, müssen kleine und mittelständische Unternehmen, die in Gefahr sind, in kaptiven Wertschöpfungsketten gefangen zu sein, aus denen ein Ausbrechen nur selbstschädigend erfolgen kann, ihr Organisationskapital erhöhen. Das betrifft zum einen Forschung und Entwicklung zu Recycling und Substitution und zum anderen den Aufbau von Kompetenzen. So hat bisher fehlendes Wissen über Schmelz- und Konzentrationsprozesse den Aufbau wettbewerbsfähiger Fabriken zur Verarbeitung schwerer Seltener Erden außerhalb Chinas beschränkt. ${ }^{94}$ Das hat umso höhere Relevanz als mangels ausreichender eigener Vorkommen und aufgrund hoher Erschließungskosten die Marktanteile in der EU zukünftig nicht in der Gewinnung, sondern in der Verarbeitung Seltener Erden und (wie bisher) ihrer Endprodukte liegen werden. So können nationale Rohstoffstrategien in der Circular Economy neu, d. h. vulnerabilitätsarm, ausgerichtet werden.

\section{Literatur}

Adger, W. Neil (2006): Vulnerability, Global Environmental Change, 16 (3), 268-281

Akamp, Marion/Mesterharm, Michael/Müller, Martin (2010): Vulnerabilitätsbezogene Wertschöpfungskettenanalyse. 1. Werkstattbericht, Juni 2010. Oldenburg: Carl von Ossietzky Universität Oldenburg

Amador, João (2018): Who's Who in Global Value Chains? A Weighted Network Approach. Open Economies Review, 29 (5), 1039-1059

Baldwin, Richard (2013): Global supply chains. Why they emerged, why they matter, and where they are going. In: Elms, Deborah K./Low, Patrick,Hrsg.: Global Value chains in a changing world. Genf: WTO Publications, 13-59

Baumol, William J./Lee, Kyu Sik (1991): Contestable Markets, Trade, and Development, The World Bank Research Observer, 6 (1), 1-17

Blum, Ulrich/Bahke, Thorsten/Eickhoff, Gisela (2002): Normen und Wettbewerb. Berlin: Beuth Verlag

94 Keilhacker/Minner 2017, 360.
Blum, Ulrich (2018): Der Kampf um Wertschöpfungsketten: Krieg gegen den Freihandel? Wirtschaftsdienst, 98 (10), 737-743

Blum, Ulrich (2019): Die neue Geoökonomik der Seidenstraße als globaler Handelsweg, in Blum, Ulrich,Hrsg.: Handelsstraßen zwischen Kooperation und Konflikt. Halle/Saale: Lehrstuhl für Wirtschaftspolitik und Wirtschaftsforschung, Martin-LutherUniversität Halle-Wittenberg, Series in Political Economy and Economic Governance, 7-27

Blum, Ulrich (2020): Wirtschaftskrieg. Rivalität ökonomisch zu Ende denken. Wiesbaden: Springer

Blum, Ulrich (2021): Kreislaufführung von Werkstoffen, Komponenten und Produkten: eine ökonomische Herausforderung, Wirtschaftsdienst, 101 (3), 186-193

Blum, Ulrich/Schmid, Marc (2015): 可再生能源和战略资源的瓶 颈 (Erneuerbare Energien und Engpässe bei strategischen Ressourcen), Annual Report on China's Low-Carbon Economic Development (2015). Blue Book of Low-Carbon Economy. Beijing: Social Sciences Academic Press, 169-180

Blum, Ulrich/Wehrspohn, Ralf B., Hrsg. (2021): Recycling, Substitution, Design und Nachhaltigkeit: Neue Wege der Werkstoffökonomik. Halle/Saale: Lehrstuhl für Wirtschaftspolitik und Wirtschaftsforschung, Martin-Luther-Universität Halle-Wittenberg, Series in Political Economy and Economic Governance 15

Braido, Luis H. B./Shalders, Felipe L. (2015): Monopoly rents in contestable markets, Economics Letters, 130, 89-92

Bugert, Niels/Lasch, Rainer (2018): Supply chain disruption models. A critical review, Logistics Research, 11 (5), 1-35

Bundesagentur für Arbeit (2020): Auswirkungen der Corona-Krise auf den Arbeits- und Ausbildungsmarkt, Berichte: Arbeitsmarkt kompakt, November 2020, http://www.am-kompakt-coronad-0-202010-pdf.pdf (arbeitsagentur.de) (letzter Abruf 27.06.2021).

Cantner, Uwe (2007): Firms' Differential Innovative Success and Market Dynamics. Jena: Universität Jena (Jena Economic Research papers \# 2007 - 078)

Cantner, Uwe/Savin, Ivan/Vannuccini, Simone (2019): Replicator dynamics in value chains: explaining some puzzles of market selection, Industrial and Corporate Change, 28 (3), 589-611, https://doi.org/10.1093/icc/dty060

Carpenter, Ryan P. (2012): The bottom of the smart weapon production chain: Securing the supply of rare earth elements for the U. S. military, Public Contract Law journal, 41 (2), 411-434

Deubel, Marco/Moormann, Jürgen/Holotiuk, Friedrich (2017): Nutzung der Blockchain-Technologie in Geschäftsprozessen, in: Eibl, Maximilian/Gaedke, Martin,Hrsg.: INFORMATIK 2017, Lecture Notes in Informatics (LNI), Gesellschaft für Informatik, Bonn, 829-842

Deutsche Bundesbank (2018): Zu den Auswirkungen der Internationalisierung deutscher Unternehmen auf die inländische Investitionstätigkeit, Monatsbericht, Januar 2018 , 13-27

Dollar, David/Kidder, Matthew (2017): Institutional quality and participation in global value chains, in: International Bank for Reconstruction and Development/The World Bank et al., Hrsg.: Global value chain development report 2017. Measuring and analyzing the impact of GVCs on economic development. Washington: World Bank Publications, 161-173. 
Engelhardt-Nowitzki, Corinna et al. (2010): Vorwort, in: EngelhardtNowitzki, Corinna et al.,Hrsg.: Supply Chain Network Management. Gestaltungskonzepte und Stand der praktischen Anwendung. Wiesbaden: Gabler V-X

Enyinda, Chris I./Szmerekovsky, Joseph (2008): Sense and Respond Supply Chain: A prescription for mitigating vulnerability in the US pharmaceutical value chain, The Journal of Global Business Issues, 2 (2), 95-103

Escaith, Hubert (2017): Accumulated trade costs and their impact on domestic and international value chains, in: International Bank for Reconstruction and Development/The World Bank et al., Hrsg.: Global value chain development report 2017. Measuring and analyzing the impact of GVCs on economic development. Washington: World Bank Publications, 97-117

Escaith Hubert/Inomata Satoshi (2016): The Evolution of Industrial Networks in East Asia: Stylized Facts and Role of Trade Facilitation Policies. In: Wignaraja G., Hrsg.: Production Networks and Enterprises in East Asia. ADB Institute Series on Development Economics. Tokyo: Springer, 113-138

Gereffi, Gary et al. (2005): The governance of global value chains, Review of International Political Economy, 12 (1), 78-104

Gereffi, Gary (2011): Global Value Chains and International Competition, Antitrust Bulletin, 56 (1), 37-56

Gereffi, Gary/Lee, Joonkoo (2012): Why the world suddenly cares about global supply chains, Journal of Supply Chain Management, 48 (3), 24-32

Gibbon, Peter/Henriksen, Lasse Folke (2011): On the pre-history of ISO 9000: The making of a neo-liberal standard. In: Ponte, Stefano/Gibbon, Peter/Vestergaard, Jakob,Hrsg.: Governing through standards. Origins, drivers and limitations. Chippenham/Eastbourne: Palgrave Macmillan, 130-158

Gleißner, Werner (2001): Identifikation, Messung und Aggregation von Risiken, in: Gleißner, W.; Meier, G.,Hrsg.: Wertorientiertes Risikomanagement für Industrie und Handel. Wiesbaden: Gabler-Verlag, 111-137

Gleißner, Werner (2018): Risiko, Volkswirtschaft und Wohlstand, in: Growitsch, C./Loose, S./Wehrspohn, R. B.,Hrsg.: Beiträge zu Wirtschaftspolitik und -forschung. Festschrift anlässlich der Emeritierung von Prof. Dr. Dr. h. c. Ulrich Blum. Halle/Saale: Universität Halle-Wittenberg, 55-68

Glöser-Chahoud, Simon/Tercero Espinoza, Luis (2015): Dynamische Materialfluss-Analyse von Neodym und Dysprosium als Magnetwerkstoffe. AP11: Stoffströme, Märkte und Umwelt. Dokumentation der globalen und deutschlandweiten Neodymund Dysprosium-Stoffstrommodellen. Fraunhofer-Institut für System- und Innovationsforschung (ISI); https://www. isi.fraunhofer.de/content/dam/isi/dokumente/ccn/2016/ KSE-Dynamische-Materialfluss-Analyse.pdf (letzter Abruf am 27.06.2021)

Grossman, G. M./Rossi-Hansberg, E. (2012): Task Trade between Similar Countries, Econometrica, 80 (2), 593-562

Hazzan, Michael J./Jones, Irene (2004): A Graphical Method for Planning Security Vulnerability Analyses of Transportation \& Value-Chain Activities. Center for Chemical Process Safety 19th Annual International Conference, Emergency Planning: Preparedness, Prevention \& Response, 29.-30. Juni und 1. Juli 2004, Orlando/Florida. Center for Chemical Process Safety of the American Institute of Chemical Engineers, Hrsg. (2004): CCPS Conference Proceedings, New York, 263-276
Heckmann, Iris/Comes, Tina/Nickel, Stefan (2015): A critical review on supply chain risk. Definition, measure and modeling, Omega, 52, 119-132

Hult, G. Tomas M. et al. (2010): Risk uncertainty and supply chain decisions, Decision Sciences, 41 (3), 435-458

Inomata, Satoshi (2017): Analytical frameworks for global value chains. An overview. In: International Bank for Reconstruction and Development/The World Bank et al.,Hrsg.: Global value chain development report 2017. Measuring and analyzing the impact of GVCs on economic development. Washington: World Bank Publications, 15-35

International Organization for Standardization (ISO) (2016): Strategic business plan ISO/TC 298.10/28/2016. Version: Draft \#2, http://www.ISO_TC_298__Rare_earth_.pdf (letzter Abruf am 6.8.2018).

Jüttner, Uta/Maklan, Stan (2011): Supply chain resilience in the global financial crisis. An empirical study, Supply Chain Management. An International Journal, 16 (4), 246-259

Keilhacker, Michael L./Minner, Stefan (2017): Supply Chain risk management for critical commodities: A system dynamics model for the case of the rare earth elements, Resources, Conservation and Recycling, 125, 349-362

Klooster, Dan (2011): The local instrumentality of global standards. How Mexican indigenous communities use FSC certification to foster a furniture production network, in: Ponte, Stefano/ Gibbon, Peter/Vestergaard, Jakob,Hrsg.: Governing through standards. Origins, drivers and limitations. Chippenham/ Eastbourne: Palgrave Macmillan, 266-288

Krugman, Paul (1994): Competitiveness: A Dangerous Obsession, Foreign Affairs, 73, 28-44

Labucay, Inéz (2018): Materials Data Space ${ }^{\circledR}$ and Standardization. A prerequisite for materials-intensive production and sustainable development? in: Blum, Ulrich,Hrsg.: Economic Governance und Ordonomik. Halle/Saale: Lehrstuhl für Wirtschaftspolitk und Wirtschaftsforschung, Martin-Luther-Universität Halle-Wittenberg, Series in Political Economy and Economic Governance 11, 183-191

Labucay, Inéz (2021): Standardisierung in der Kreislaufwirtschaft. Vom Material zur Wertschöpfungskette, in: Blum, Ulrich/ Wehrspohn, Ralf B.,Hrsg.: Recycling, Substitution, Design und Nachhaltigkeit: Neue Wege der Werkstoffökonomik. Halle/Saale: Lehrstuhl für Wirtschaftspolitk und Wirtschaftsforschung, Martin-Luther-Universität Halle-Wittenberg, Series in Political Economy and Economic Governance 15

Lemley, Mark A. (2008): Ignoring patents, Michigan State Law Review, 19 (Spring), 19-34

Lessard, Donald (2013): Uncertainty and risk in global supply chains, in: Elms, Deborah K./Low, Patrick,Hrsg.: Global Value chains in a changing world. Genf: WTO Publications, 195-220

Machacek, Erika/Fold, Nils (2018): Competitors contained? Manufacturer strategies in the global rare earth value chain: Insights from the magnet filament, The Extractive Industries and Society 5(1), 18-27

Meinert, Kathrin/Müller, Holger (2018): Blockchains im Supply Chain Management 4.0. Leipzig: Hochschule für Technik, Wirtschaft und Kultur (HTWK); https://fww.htwk-leipzig. de/fileadmin/portal/f_wiwi/fakultaet/personen/PROF/ mueller_h_scm/Studie_Blockchains_fuer_das_Supply_Chain_ Management_4.pdf 
Min, Hokey (2019): Blockchain technology for enhancing supply chain resilience, Business Horizons, 62, 35-45

Nadvi, Khalid (2008): Global standards, global governance and the organization of global value chains, Journal of Economic Geography, 8(3), 323-343

Navaz, Waqas/Linke, Patrick/Koç, Muammar (2019): Safety and sustainability nexus. A review and appraisal, Journal of Cleaner Production, 216 (4), 74-87

Neureuther, Brian D./Kenyon, George (2009): Mitigating Supply Chain Vulnerability, Journal of Marketing Channels, 16 (3), 245-263

Peck, Helen (2005): Drivers of supply chain vulnerability: an integrated framework, International Journal of Physical Distribution \& Logistics Management, 35 (4), 210-232

Pei, J. (2018): Gaokao Reform and China's Upgrading in GVCs, Vortrag im Rahmen des CEM-Workshop on Total Design Management and Global Value Chains, Halle, mimeo

Pell, Robert et al. (2019): Temporally explicit life cycle assessment as an environmental performance decision-making tool in rare earth project development, Minerals Engineering, 135, 64-73

Ponte, Stefano/Gibbon, Peter/Vestergaard, Jakob (2011): Governing through standards: An introduction, in: Ponte, Stefano/Gibbon, Peter/Vestergaard, Jakob,Hrsg.: Governing through standards. Origins, drivers and limitations. Chippenham/Eastbourne: Palgrave Macmillan, 1-24

Prahalad, Coimbatore Krishnarao/Hamel, Gary (1990): The core competence of the organization, Harvard Business Review, 90 (3), 78-90

Quoreshi, Shahiduzzaman/Stone, Trudy-Ann (2019): Do Global Value Chains Make Firms More Vulnerable To Trade Shocks? Evidence from Manufacturing Firms in Sweden, Journal of Risk and Financial Management, 12(3), 151-166

Runde, Daniel F./Bandura, Romina/Hammond, MacKenzie (2019): Making the Future Work for Us. Technological Impacts on Labor in the Developing World, in: Aaron Milner/Erol Yayboke (Hrsg.): Beyond Technology. The Fourth Industrial Revolution in the Developing World. Washington, D.C.: Center for Strategic and International Studies, 33-39

Salop, Steven C./Scheffman, David T. (1983): Raising Rivals' Costs, The American Economic Review, 73(2), 267-271

Schacht, Hjalmar (1937): Germany's Colonial Demands, Foreign Affairs, 16 (2), 223-234

Scheer, August-Wilhelm (2013): Die vierte industrielle Revolution zeichnet sich ab! Im Gespräch mit Professor Dr. Dr. h. c. mult. Wolfgang Wahlster, in: Scheer, A.-W.,Hrsg.: Industrie 4.0. Wie sehen Produktionsprozesse im Jahr 2020 aus? Saarbrücken: IMC AG, 7-11

Schumpeter, Joseph (1912): Theorie der wirtschaftlichen Entwicklung. Berlin: August Rabe

Schumpeter, Joseph (1927): Unternehmer, in: Handwörterbuch der Staatswissenschaften. Vierte Auflage, Band VIII, Jena: Gustav FischerVerlag, 476-487.

Schumpeter, Joseph (1942): Capitalism, Socialism and Democracy. New York: Routledge

Stindt, Dennis/Sahamie, Ramin (2014): Review of research on closed loop supply chain management in the process industry, Flexible and Service Manufacturing Journal, 26 (1-2), 268-293

Svensson, Göran (2000): A conceptual framework for the analysis of vulnerability in supply chains, International Journal of Physical and Distribution and Logistics Management, 30 (9), 731-749
Tewari, Anurag (2013): Upstream Supply Chain Vulnerability, Robustness and Resilience: A Systematic Review of Literature. Cranfield: Cranfield University, Dissertation

Töpfer, Armin/Blum, Ulrich/Blind, Knut (2000): Gesamtwirtschaftlicher Nutzen der Normung: Zusammenfassung der Ergebnisse. Wissenschaftlicher Endbericht mit praktischen Beispielen. Berlin u. a.: Beuth Verlag

Tomer, John F. (2008): Intangible capital. Its contribution to economic growth, well-being and rationality. Cheltenham, UK/ Northampton, MA: Edward Elgar.

UNCOMTRADE (o.D.): Seltene Erden (HS-commodity codes 280530 und HS-2846), unter der Adresse https://comtrade.un.org/ data/, (letzter Abruf am 19.6. 2019

United Nations Environment Programme - UNEP/European Patent Office - EPO (2015): Climate change mitigation technologies in Europe - evidence from patent and economic data. Nairobi/ Munich: Grantham Research Institute on Climate Change/ London School of Economics. Climate change mitigation technologies in Europe - evidence from patent and economic data (epo.org) (letzter Abruf am 27.06.2021).

United States Department of Agriculture USDA (2019): Good Agricultural Practices (GAP) and Good Handling Practices (GHP). Washington, D.C.: USDA; https://www.ams.usda.gov/ services/auditing/gap-ghp

Vidal, 0./Goffé, B./Arndt, N. (2013): Metals for a Low-Carbon Society, Nature Geoscience, 6, 894-896

Voncken, Jack H. L. (2016): The rare earth elements. An introduction. SpringerBriefs in Earth Sciences. Dordrecht: Springer Nature

Wagner, Stephan M./Bode, Christoph (2006): An empirical investigation into supply chain vulnerability, Journal of Purchasing \& Supply Management, 12 (6), 301-312

Wagner, Stephan M. et al. (2010): Supply Chain Risikomanagement: Besonderheiten und Herausforderungen für kleine und mittlere Unternehmen, in: Engelhardt-Nowitzki, Corinna et al.,Hrsg.: Supply Chain Network Management. Gestaltungskonzepte und Stand der praktischen Anwendung. Wiesbaden: Gabler, 97-116

Wagner, Stephan M./Neshat, Nikrouz (2012): A comparison of supply chain vulnerability indices for different categories of firms, International Journal of Production Research, 50 (11), 2877-2891

Walachowicz, Frank et al. (2014): Verbundprojekt: Recycling von Elektromotoren - MORE. Teilprojekt: Ökobilanz der Recyclingverfahren. Projekt gefördert im Rahmen des Programms „Schlüsseltechnologien für die Elektromobilität (STROM)“ des BMBF. Berlin/Darmstadt: Öko-Institut e. V./SIEMENS; https:// www.oeko.de/fileadmin/oekodoc/MORE-LCA-Endbericht.pdf

Walrave, Bob/Raven, Rob (2016): Modelling the dynamics of technological innovation systems, Research Policy, 45, 1833-1844

Wehrspohn, Ralf (2017): Leitprojekt “Kritikalität Seltener Erden“. Hightech-Rohstoffe: Gewinnen und Ersetzen, in: Neugebauer, R.,Hrsg.: Ressourceneffizienz. Schlüsseltechnologien für Wirtschaft und Gesellschaft. Berlin: Springer Vieweg, 301-319

Williamson, Oliver, E. (2005): The Economics of Governance, American Economic Review 95 (2), 1-18

Williamson, Oliver E. (2010): Transaction Cost Economics: The Natural Progression, The American Economic Review, 100 (3), $673-690$

Wolter, Marc Ingo et al. (2016): Wirtschaft 4.0 und die Folgen für Arbeitsmarkt und Ökonomie. Szenario-Rechnungen im Rahmen der BIBB-IAB-Qualifikations- und Berufsfeldprojektionen. 
Nürnberg: Institut für Arbeitsmarkt- und Berufsforschung der Bundesagentur für Arbeit, IAB-Forschungsbericht 13/2016 World Intellectual Property Organization (2017): Smartphones: What's Inside the Box?, Chapter 4, in: World Intellectual Property Report 2017 - Intangible Capital in Global Value Chains, Genf: World intellectual Property Organization
Yüzgülec, Gökhan/Witthaut, Markus/Hellingrath, Bernd (2010): Auswirkungen des Information Sharings in Supply Chains, in: Engelhardt-Nowitzki, Corinna et al. (Hrsg).: Supply Chain Network Management. Gestaltungskonzepte und Stand der praktischen Anwendung. Wiesbaden: Gabler, 81-96 